\title{
钯-单膦催化剂在烯烃不对称硅氢化反应中的应用
}

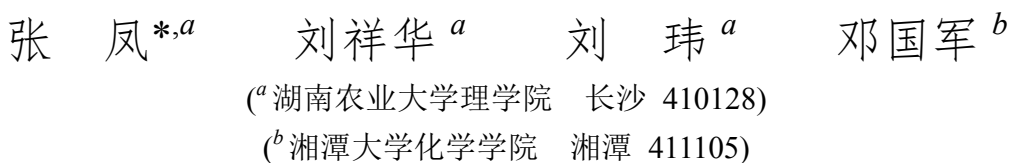

摘要 烯烃的不对称硅氢化反应作为合成手性仲醇的一种重要方法, 受到了国内外众多学者的关注. 研究表明, 钯-单 膦催化剂对该类反应有着优异的催化活性和选择性. 由于结构稳定、易于合成及修饰、催化性能独特等优点，手性单 膦配体取得了迅速的发展. 其中, 基于二茂铁骨架的平面手性膦配体、基于联芳基骨架的轴手性膦配体以及手性亚磷 酰胺酯配体在烯烃的不对称硅氢化反应中取得了优异的催化效果. 详细总结了近年来钯-单膦催化剂催化的烷基烯烃、 苯乙烯及其衍生物、1,3-二烯烃等底物的不对称硅氢化反应研究进展, 并对其发展前景进行了展望.

关键词 钯催化; 单膦配体; 烯烃; 不对称硅氢化

\section{Application of Pd-Monodentate Phosphorus Catalysts in the Asymmetric Hydrosilylation Reactions of Alkenes}

\author{
Zhang, Feng*,a Liu, Xianghua ${ }^{a} \quad{\text { Liu, } \text { Wei }^{a} \quad \text { Deng, Guojun }}^{b}$ \\ ( ${ }^{a}$ College of Science, Hunan Agricultural University, Changsha 410128) \\ $\left({ }^{b}\right.$ College of Chemistry, Xiangtan University, Xiangtan 411105)
}

\begin{abstract}
Asymmetric hydrosilylation of alkenes, which has been recognized as an important method for the preparations of optically active secondary alcohols, deserves widespread attention over the world. It is reported that such reaction can be catalyzed by Pd-monodentate phosphorus catalysts with excellent reactivity and enantioselectivity. In the past decades, a wide variety of chiral monodentate phosphorus ligands have been developed because of their stable structure, facile synthesis, convenient modification, unique efficiency. Among them, there are three predominant classes of ligands-phosphines based on an axially chiral biaryl scaffold, phosphines based on a planar chiral ferrocene scaffold and chiral phosphoramidites. Herein, the recent advances in asymmetric hydrosilylation of alkyl-substituted alkenes, styrene derivatives, 1,3-dienes and other carbon-carbon double bond compounds catalyzed by palladium monodentate phosphorus catalysts are summarized. The perspective is also discussed.
\end{abstract}

Keywords Pd catalyzed; monodentate phosphorus ligands; alkenes; asymmetric hydrosilylation

钯催化剂具有独特的性质，成为有机合成中不可或 缺的工具 ${ }^{[1,2]}$. 钯催化的烯烃的不对称反应是合成多种 手性化合物的重要方法之一 ${ }^{[3,4]}$. 手性仲醇是一类非常 重要的有机合成中间体, 广泛应用于医药、农药及食品 添加剂等合成中 ${ }^{[5]}$. 烯烃的不对称硅氢化反应是氢硅烷 对碳一碳双键的立体选择性加成, 碳-硅键经过 FlemingTamao 氧化反应在手性中心完全保持不变的前提下转 化成碳一氧键，从而成为制备手性仲醇的有效途径之一. 在合成手性仲醇的众多方法中, 钯催化的烯烃的不对称
硅氢化反应由于反应条件温和、反应效率高、环境友好、 无需耐高压设备和操作简便等优点引起了科研工作者 的普遍关注.

手性配体是手性催化剂产生不对称诱导和控制的 源泉，不对称催化反应的关键之一是手性配体的设计合 成. 虽然早在 20 世纪 70 年代, Kumada 等 ${ }^{[6]}{ }^{6}$ 就报道了首 例钯催化的烯烃的不对称硅氢化反应，但是催化剂的选 择性很低. 直到 20 世纪 90 年代初, 采用钯金属前体及 单膦配体所形成的手性配合物作为催化剂后, 烯烃的不

* Corresponding author. E-mail: zhang_feng0911@163.com
Received April 10, 2017; revised May 15,2017; published online June 7, 2017.

Project supported by the National Natural Science Foundation of China (No. 21302051) and the Natural Science Foundation of Hunan Province (No. 14JJ3091)

国家自然科学基金(No. 21302051)和湖南省自然科学基金(No. 14JJ3091)资助项目. 
对称硅氢化反应才取得了突破性的进展. 单膦配体具有 易于合成、结构灵活、催化性能优异等特点. 此外, 当 中心金属离子只能提供一个配位轨道时, 单膦配体就能 够以一个配体同中心金属离子络合，进而催化不对称反 应. 正是由于这些优点, 越来越多的单膦配体被合成出 来. 目前, 单膦配体已成为不对称催化领域中研究热点, 成功应用于不对称催化共轭加成反应、不对称催化烯丙 基取代反应、不对称催化氢化反应等 ${ }^{[7,8]}$.

单膦配体按照磷原子成键的种类主要可以分为膦 配体(phosphine)、亚磷酰胺酯配体(phosphoramidite)、亚 膦酸酯配体(phosphonite)、亚磷酸酯配体 (phosphite)以 及其他类型的单膦配体(图 1). 其中, 平面手性单齿膦配 体、轴手性单齿膦配体以及手性单齿亚磷酰胺配体在烯 烃的不对称硅氢化反应中取得了优异的催化效果. 鉴于 单膦配体对不对称催化硅氢化反应的重要性, 且对烯烃 的不对称硅氢化反应系统评述较少 ${ }^{[9]}$, 本文按照单膦配 体的结构类型, 对钯催化的烯烃的不对称硅氢加成的研 究进展加以综述, 以便于人们对该领域进行全面的了

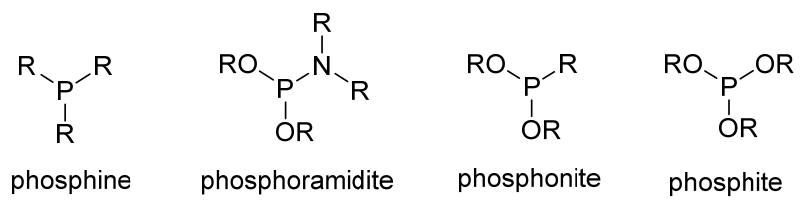

图 1 单膦配体的结构示意图

Figure 1 Structures of chiral monodentate phosphorus ligands
解，更好地设计配体及催化剂从而推进该领域的应用发 展.

\section{1 手性单齿膦配体在钯催化的烯烃的不对称硅 氢化反应中的应用}

\section{1 平面手性单齿膦配体}

在不对称催化领域中，平面手性二茂铁类配体以其 特殊的刚性骨架结构和良好的电子效应从众多手性配 体中脱颖而出. 其中, 许多含膦、氮的二茂铁配体(例如 Josiphos、Ferriphos、Walphos 等)由于兼具平面手性及 中心手性，在多种不对称催化反应中体现出了优异的不 对称诱导能力, 有着广泛的应用前景 ${ }^{[10]}$. 钯催化的不对 称硅氢化反应中, 表现较为突出的二茂铁骨架手性配体 则是单齿膦配体(图 2). 这类配体往往含有杂原子或者 芳环，与金属钯配位时，除了磷原子作为主要的配位点 外，金属与杂原子或芳基可能存在着次级相互作用. 次 级相互作用的强弱影响催化剂的活性及选择性. 目前, 平面手性的二茂铁类单膦配体已成功应用于钯催化的 苯乙烯、1,3-二烯烃等底物的不对称硅氢化反应中.

1980 年, Kumada 等 ${ }^{[11]}$ 报道了首例二茂铁骨架的单 齿膦配体在烯烃的不对称硅氢化反应中的性能研究. 他 们将配体 L1 与钯形成的络合物(Cat. 1)用于苯乙烯与三 氯硅氢的不对称硅氢化反应中，当催化剂的用量为 0.01

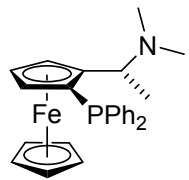

L1

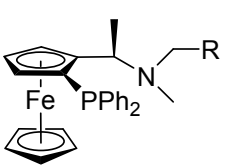

L2a R $=n-\mathrm{C}_{3} \mathrm{~F}_{7}$ L2b R $=n-\mathrm{C}_{8} \mathrm{~F}_{17}$
L3a $\mathrm{R}^{1}=\mathrm{H}, \mathrm{R}^{2}=2,4,6-\mathrm{Me}_{3} \mathrm{C}_{6} \mathrm{H}_{2}, \mathrm{Ar}=\mathrm{Ph}$ L3b R $\mathrm{R}^{1}=\mathrm{H}, \mathrm{R}^{2}=2,4,6-\mathrm{Me}_{3} \mathrm{C}_{6} \mathrm{H}_{2}, \mathrm{Ar}=3,5-\left(\mathrm{CF}_{3}\right)_{2} \mathrm{C}_{6} \mathrm{H}_{3}$

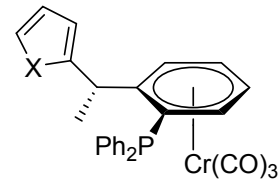

L4a $X=O$

L4b $X=S$

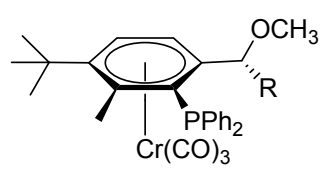

L5a $\mathrm{R}=\mathrm{Bn}$ L5b R = 3,5- $\left(\mathrm{CH}_{3}\right)_{2} \mathrm{C}_{6} \mathrm{H}_{3} \mathrm{CH}_{2}$

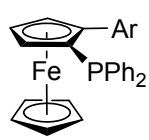

L6a $\mathrm{Ar}=4-\mathrm{MeOC}_{6} \mathrm{H}_{4}$ L6b $\mathrm{Ar}=3,5-\left(\mathrm{CF}_{3}\right)_{3} \mathrm{C}_{6} \mathrm{H}_{3}$ L6c $\mathrm{Ar}=1-\mathrm{Naphthyl}$

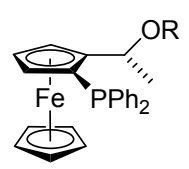

L7a $\mathrm{R}=\mathrm{CH}_{3}$ L7b $\mathrm{R}=\mathrm{CH}_{2} \mathrm{CF}_{3}$ L7c $R=A c$

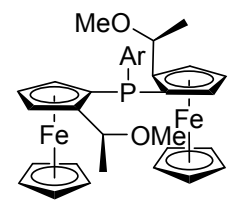

L8a $\mathrm{Ar}=\mathrm{Ph}$

L8b $\mathrm{Ar}=4-\mathrm{MeOC}_{6} \mathrm{H}_{4}$ L8c $\mathrm{Ar}=3,5-\left(\mathrm{CF}_{3}\right)_{2} \mathrm{C}_{6} \mathrm{H}_{3}$

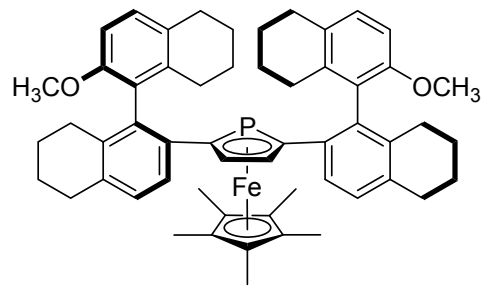

19

图 2 用于烯烃不对称硅氢化反应的平面手性膦配体

Figure 2 Planar chiral phosphines used in the asymmetric hydrosilylation of alkenes 

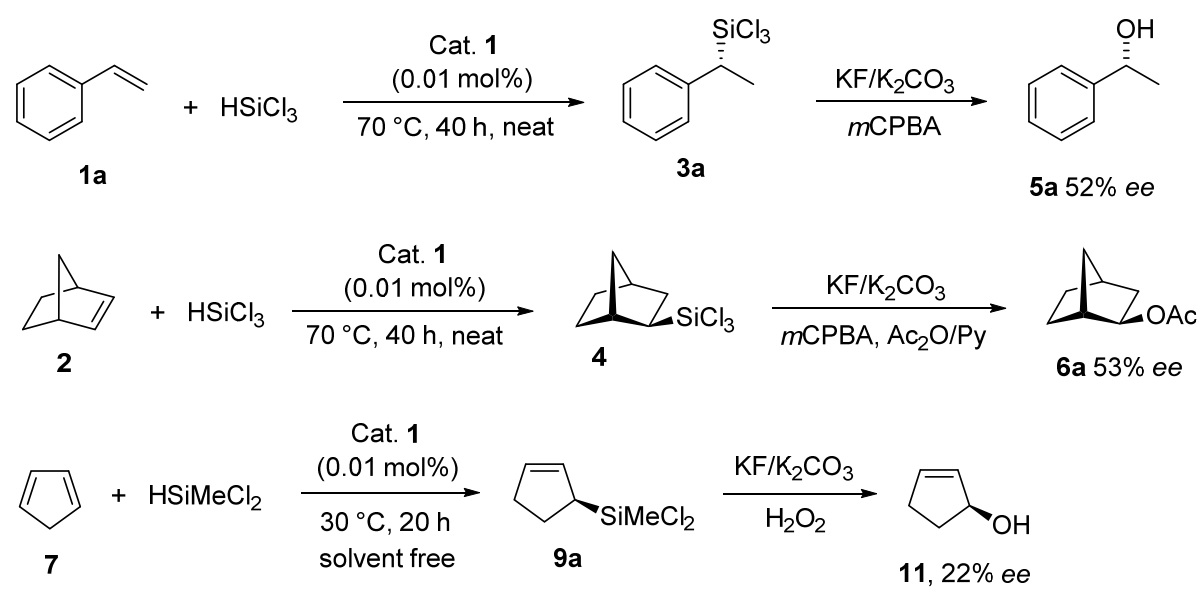

Cat. 1
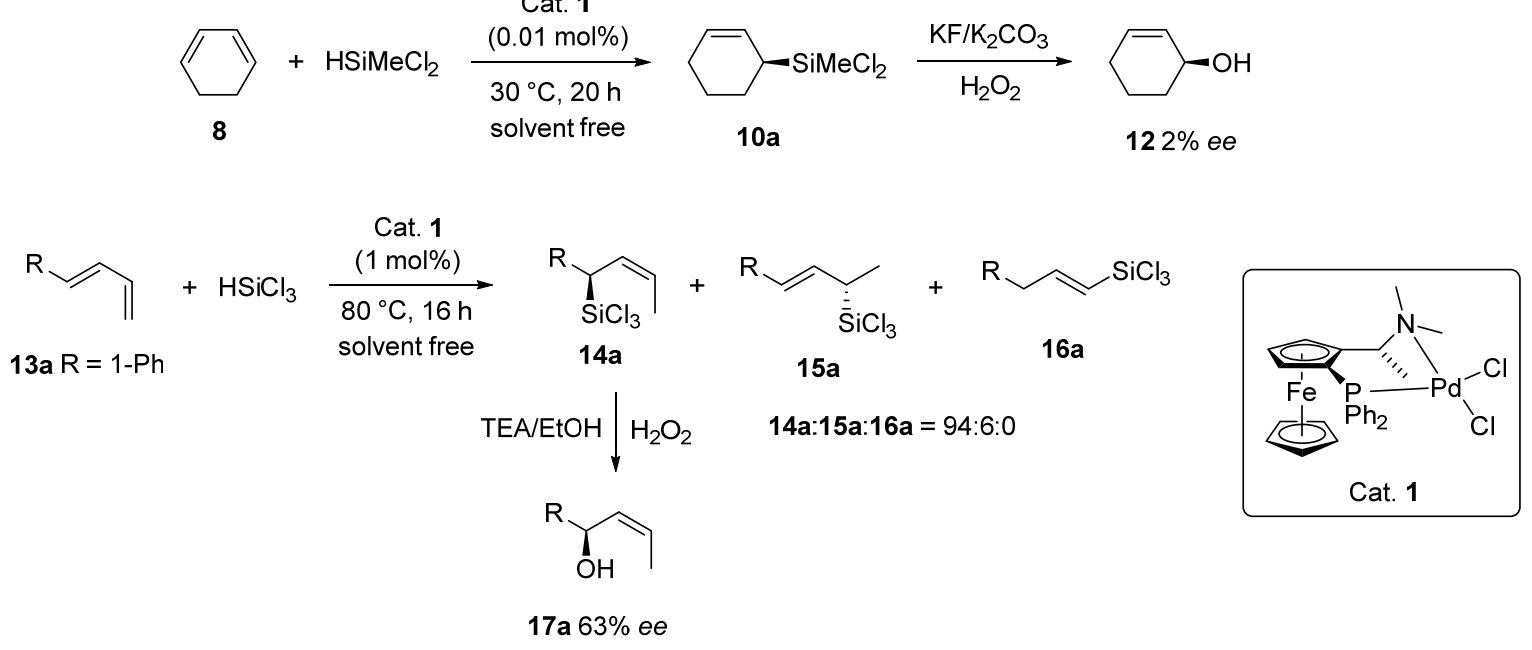

图式 1 平面手性膦配体 $\mathbf{L 1}$ 在烯烃的不对称硅氢化反应中的应用

Scheme 1 Application of planar chiral phosphine $\mathbf{L 1}$ in the asymmetric hydrosilylation of alkenes

$\mathrm{mol} \%, 70{ }^{\circ} \mathrm{C}$ 下, 反应 $40 \mathrm{~h}$, 底物才能完全转化, 所得产 物的 $e e$ 值仅为 $52 \%$ (Scheme 1). 相同条件下, 将配体 $\mathbf{L 1}$ 应用于降冰片烯的不对称硅氢化反应中, 所得产物的 $e e$ 值为 $53 \%$.

1983 年, Hayashi 等 ${ }^{[12]}$ 报道了首例环状 1,3-二烯烃 的不对称硅氢化反应. 环状 1,3-二烯烃与硅烷的不对称 硅氢化反应以 1,4-顺式加成为主生成手性烯丙基硅烷. 烯丙基硅烷通过 Fleming-Tamao 氧化反应进一步生成 手性烯丙醇. 他们将一系列二茂铁骨架的单齿膦配体应 用于钯催化的环戊二烯及 1,3 -环己二烯的不对称硅氢化 反应中. $30{ }^{\circ} \mathrm{C}$ 下, Cat. 1 在环戊二烯与甲基二氯硅烷的 反应中, 以 $87 \%$ 的产率得到 $e e$ 值为 $22 \%$ 的产物. 当底 物为 1,3-环已二烯时, 虽然产率高达 95\%, 产物的 ee 值 仅为 $2 \%$. 随后，他们 ${ }^{[13]}$ 将二茂铁骨架的催化剂 Cat. 1 应 用于钯催化的链状 1,3-二烯烃如 1-苯基-1,3-丁二烯(13a) 的不对称硅氢化反应中, 以 $62 \%$ 的收率得到了两个产 物. 其中, 1,4-加成产物和 1,2-加成产物的比例为 $94: 6$, $e e$ 值分别为 $63 \%$ 和 $30 \%$.
接下来，他们 ${ }^{[14]}$ 又合成了含氟长烷基链的配体 $\mathbf{L 2}$, 并将其与金属钯形成的络合物 Cat. 2 应用于环戊二烯的 不对称硅氢化反应中 (Scheme 2). 当催化剂的用量为 $0.016 \mathrm{~mol} \%$, 室温下反应 90 h, Cat. 2a 和 Cat. 2b 所得产 物的 $e e$ 值分别为 $57 \%$ 和 $55 \%$. 而相同条件下, Cat. 1 在 该反应中没有活性. 这可能是含氟侧链的引入使得催化 剂溶解性能有了很大提高，同时大大降低了氮原子的配 位能力，便于底物与金属配位，从而促进催化反应的进 行. 然而, 含氟长烷基链的配体 $\mathbf{L} 2$ 在链状 1,3-二烯烃的 不对称硅氢化反应中, 选择性仍然不理想.

1998 年, Togni 等 ${ }^{[15]}$ 将含有吡唑环的二茂铁骨架的 单膦配体 $\mathbf{L 3}$ 与金属钯形成的络合物 Cat. 3 应用于一系 列苯乙烯及其衍生物的不对称硅氢化反应中, 虽然催化 剂的活性略有提高, 但是仅取得了中等选择性(Scheme 3). 同时，他们发现产物的构型与底物中对位基团的电 子效应有关. 例如, Cat. 3a 催化的富电子的 4-氨基苯乙 烯得到 $R$ 构型为主的产物, $e e$ 值为 $64 \%$; 而缺电子的 4氯苯乙烯得到 $S$ 构型的产物, $e e$ 值为 $67 \%$. 同时, 他们将 


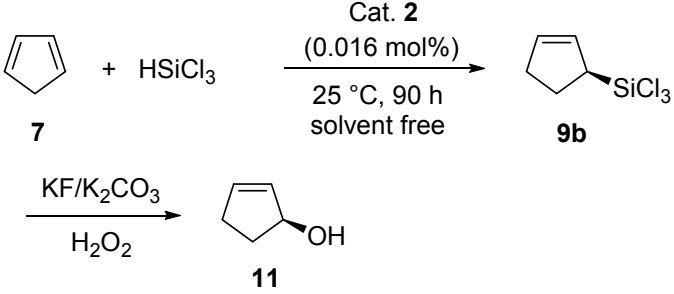

Cat. 1: no reaction

Cat. 2a: $73 \%$ yield of 9 b, $57 \%$ ee of $\mathbf{1 1}$

Cat. 2b: $41 \%$ yield of $\mathbf{9 b}, 55 \%$ ee of 11

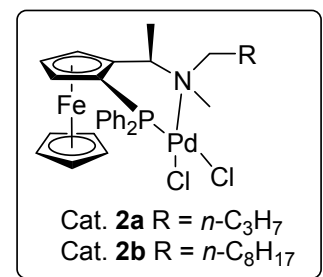

图式 2 平面手性膦配体 $\mathbf{L 2}$ 在环戊二烯的不对称硅氢化反应 中的应用

Scheme 2 Application of planar chiral phosphines L2 in the asymmetric hydrosilylation of cyclopentadiene
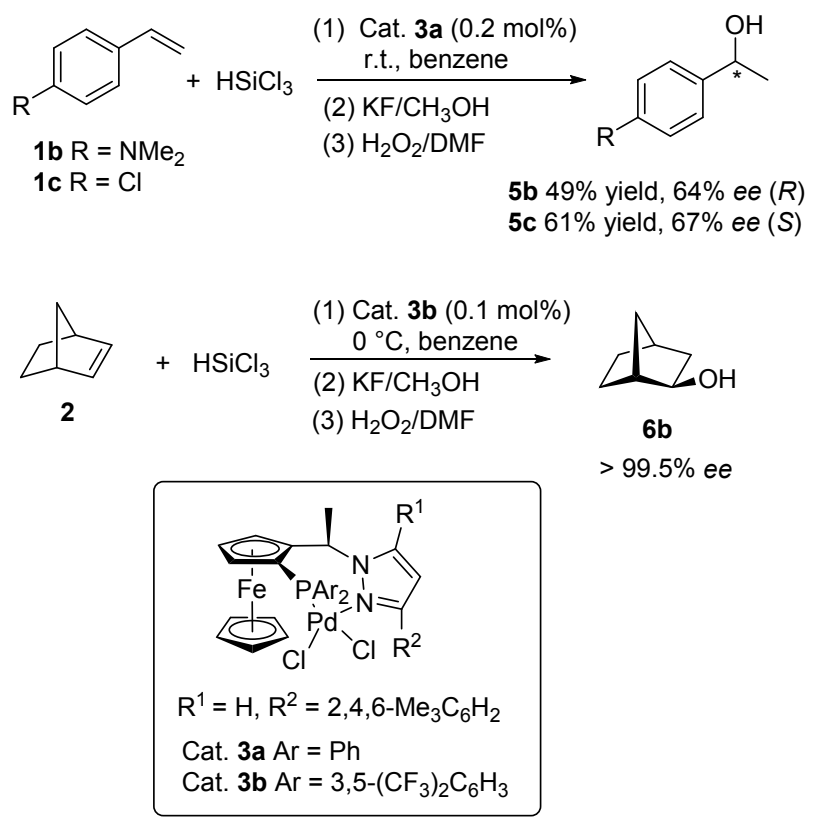

图式 3 平面手性膦配体 $\mathbf{L 3}$ 在取代苯乙烯及降冰片烯的不对 称硅氢化反应中的应用

Scheme 3 Application of planar chiral phosphines $\mathbf{L 3}$ in the asymmetric hydrosilylation of substituted styrenes and norbornene

Cat. 3 应用于 $\operatorname{Pd}(\mathrm{COD}) \mathrm{Cl}_{2}$ 催化的降冰片烯的不对称硅 氢化反应中. 研究结果表明, 催化剂的活性和选择性取 决于磷及吡唑环上的取代基. 增大 $\mathrm{R}^{2}$ 的位阻可能削弱 了氮原子的配位能力, 从而使得催化剂的活性增加, 反 应能在较低温下进行, 选择性也相应的增加. 当 $\mathrm{R}^{1}$ 为氢 原子时, 降低磷原子电子云密度, 催化剂的活性和选择 性进一步增加. 配体 $\mathbf{L 3 b}$ 的 $\mathrm{R}^{1}$ 为氢原子, 磷原子上的
芳基为吸电子的 3,5-二三氟甲基苯基, $\mathrm{R}^{2}$ 为大位阻基 2,4,6-三甲基苯基. 该配体在该降冰片烯的不对称硅氢 化反应中取得了 $>99.5 \%$ 的对映选择性. 这也是迄今为 止，该底物取得的最高对映选择性.

2001 年, Jones 等 ${ }^{[16]}$ 设计合成了一类含有杂环的新 型平面手性膦配体 L4. 采用 $\left[\mathrm{Pd}\left(\mathrm{C}_{3} \mathrm{H}_{5}\right) \mathrm{Br}\right]_{2}$ 作为催化剂 前体，催化剂用量为 $0.25 \mathrm{~mol} \%$, 并将反应体系降至 $-40{ }^{\circ} \mathrm{C}$, 含有呋喃环的配体 L4a 在苯乙烯及其衍生物 的不对称硅氢化反应中取得了 $87 \%$ ee (表 1, Entry 1). 将反应温度降至 $-50{ }^{\circ} \mathrm{C}$, 其他条件保持不变, 含噻吩 环的配体 L4b 获得了 $92 \%$ ee 的产物(表 1, Entry 2). 2006 年, Gibson 等 ${ }^{[17]}$ 将相同手性骨架的配体 L5 用于钯 催化的苯乙烯类底物的不对称硅氢化反应中，仅取了中 等的选择性(表 1, Entries 3, 4).

表 1 平面手性膦配体 L4、L5 及 L6 在钯催化的苯乙烯硅氢 化反应中的应用 ${ }^{a}$

Table 1 Application of planar chiral phosphines L4、L5 and L6 in the asymmetric hydrosilylation of styrene

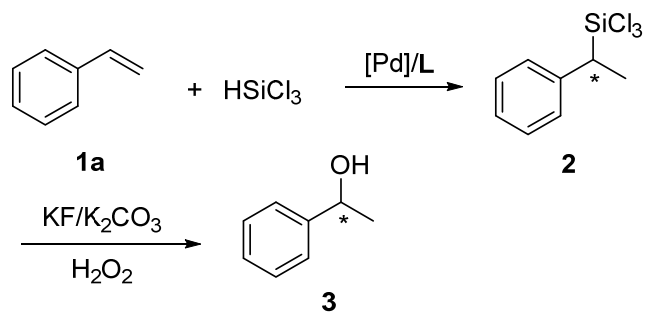

\begin{tabular}{llllcc}
\hline Entry & $\mathbf{L}$ & $\begin{array}{c}\text { Catalyst/ } \\
\text { mol\% }\end{array}$ & \multicolumn{1}{c}{ Condition } & $\begin{array}{c}\text { Yield/\% } \\
\text { of } \mathbf{2}\end{array}$ & $\begin{array}{c}\text { ee/\% } \\
\text { of 3 }\end{array}$ \\
\hline $1^{b, c, d}$ & L4a & 0.25 & $-40{ }^{\circ} \mathrm{C}, 48 \mathrm{~h}$ & $>85$ & 87 \\
$2^{b, c, d}$ & $\mathbf{L 4 b}$ & 0.25 & $-50{ }^{\circ} \mathrm{C}, 48 \mathrm{~h}$ & $>85$ & 92 \\
$3^{e}$ & $\mathbf{L 5 a}$ & 0.5 & r.t., $48 \mathrm{~h}$ & 99 & 55 \\
$4^{f}$ & $\mathbf{L 5 b}$ & 0.5 & r.t., $48 \mathrm{~h}$ & 97 & 77 \\
5 & L6a & 1 & r.t., $1.6 \mathrm{~h}$ & 100 & 86 \\
6 & L6b & 1 & r.t., $8 \mathrm{~h}$ & 100 & 25 \\
7 & L6c & 1 & r.t., $1.5 \mathrm{~h}$ & 100 & 79 \\
8 & L6b & 0.1 & r.t., $8 \mathrm{~h}$ & 100 & 90 \\
9 & L6c & 0.1 & r.t., $3.5 \mathrm{~h}$ & 100 & 85 \\
$10^{g}$ & L6c & 0.1 & r.t., $20 \mathrm{~s}$ & 100 & 62 \\
\hline
\end{tabular}

${ }^{a}$ All reactions were conducted with $\left[\mathrm{Pd}\left(\mathrm{C}_{3} \mathrm{H}_{5}\right) \mathrm{Cl}\right]_{2}$ as precursor, $[\mathbf{L}] /[\mathrm{Pd}]=2$, solvent free unless otherwise stated. ${ }^{b}[\mathbf{L}] /[\mathrm{Pd}]=1 .{ }^{c} \mathrm{CH}_{2} \mathrm{Cl}_{2}$ or $\mathrm{CDCl}_{3}$ as solvent. ${ }^{d}\left[\mathrm{Pd}\left(\mathrm{C}_{3} \mathrm{H}_{5}\right) \mathrm{Br}\right]_{2}$ as precursor. ${ }^{e} \mathrm{CH}_{2} \mathrm{Cl}_{2}$ as solvent. ${ }^{f}$ Benzene as solvent. ${ }^{g}[\mathbf{L}][[\mathrm{Pd}]=1.2$.

Johannsen 等 ${ }^{[18]}$ 将二茂铁骨架芳基修饰的配体 L6 应用于苯乙烯类底物的不对称催化反应中。他们发现增 大配体中芳环上的电子云密度, 有利于催化剂活性和选 择性的提高. 他们采用 $\left[\mathrm{Pd}\left(\mathrm{C}_{3} \mathrm{H}_{5}\right) \mathrm{Cl}\right]_{2}$ 作为催化剂前体, 配体与金属钯的比例为 2 , 催化剂的用量为 $1 \mathrm{~mol} \%$, 富 电子配体 L6a 在 $1.6 \mathrm{~h}$ 内底物完全转化, 取得了 $86 \%$ 的 对映选择性(表 1, Entry 5); 而在相同条件下，缺电子配 体 L6b 需要 $8 \mathrm{~h}$ 才能完成反应, $e e$ 值仅为 $25 \%$ (表 1 , Entry 6). 保持配体与金属的比例不变, 将催化剂的用量降为 
$0.1 \mathrm{~mol} \%$ 时, 配体 L6a 所得产物的 ee 值升高至 $90 \%$ (Entry 8), 配体 L6c 所得产物的 ee 值也由 79\% (表 1, Entry 7)升高至 85\%(表 1, Entry 9). 此外, 他们以配体 L6c 为例, 研究了配体与金属的比例对于催化剂的活性 和选择性的影响. 发现降低配体与金属的比例, 催化剂 的选择性下降明显, 但是活性却大大的增加了. 如当金 属与配体的比例为 $0.1: 0.12$ 时, 底物在 $20 \mathrm{~s}$ 内完全转 化, 转化频率(TOF)值高达 $180000 \mathrm{~h}^{-1}$ (表 1, Entry 10).

在 Chalk-Harrod 机理的基础上, 他们以实验结果为 依据, 提出了硅氢化反应可能经历了两个不同的催化循 环过程(Scheme 4): 配体与金属的比例为 2 时, 反应按 慢催化循环方向进行; 当金属与配体的比例小于 2 时, 反应往快催化循环方向进行. 在慢催化循环中, $\mathrm{PdP}_{2}$ 先 与 $\mathrm{HSiCl}_{3}$ 氧化加成, 单膦配体再与苯乙烯发生配位交 换, 烯烃插入 $\mathrm{Pd}-\mathrm{H}$ 键后, 另一个单膦配体与钯络合物 配位, 还原消除后重新生成 $\mathrm{PdP}_{2}$. 与慢循环不同的是, 当单膦配体比例较小时, 为了维持配位稳定性, 快循环 中另一个苯乙烯与钯络合物配位, 再经过还原消除得到 二配位的化合物, 最后与 $\mathrm{HSiCl}_{3}$ 氧化加成完成催化循 环. 快循环中, 第二个烯烃参与配位, 故能很好地解释 配体与金属比例降低时, 反应速率反而大大增加.
Ohmura 等 ${ }^{[19]}$ 将含氧侧链的二茂铁骨架的手性单膦 配体 L7 应用于 1,3-环己二烯的不对称硅氢化反应中 (Scheme 5). 为了提高催化剂的的活性及选择性, 他们 对配体、硅烷等进行了优化. 室温下，以 $\mathrm{Pd}(\mathrm{Ph}-\mathrm{CN})_{2} \mathrm{Cl}_{2}$ 为催化剂前体, 采用缺电子基的苯基二氟硅烷与环己二 烯反应，催化剂的用量为 $1 \mathrm{~mol} \%$ 时，含氮侧链的配体 $\mathbf{L 1}$ 仅取得了 $9 \% \mathrm{ee}$, 而含氧侧链的配体选择性有了大大 的提高. 例如, 在相同的条件下, L7a 取得了 $54 \% \mathrm{ee}$. 缺 电子配体 $\mathbf{L 7 b}$ 取得了 $65 \%$ ee. 采用 $\left[\mathrm{Pd}\left(\mathrm{C}_{3} \mathrm{H}_{5}\right) \mathrm{Cl}\right]_{2}$ 作为催 化剂前体，含吸电子基团配体 L7c ee 值升高至 77\%.上 述研究结果表明, 配体侧链上的取代基影响催化剂的活 性及选择性, 这可能与含氧侧链的配体与钯共配位能力 较弱有关.

2002 年 Hayashi 等 $^{[20]}$ 将磷上连有两个二茂铁基的含 氧侧链配体 L8 应用于 1, 3-癸二烯(13b) 的不对称硅氢化 反应中(Eq. 1). 他们也发现产物的 $e e$ 值取决于配体的电 子效应, 含缺电子基的配体往往能得到更高的 $e e$ 值. 例 如, $20{ }^{\circ} \mathrm{C}$ 下, 富电子配体 $\mathbf{L 8 b}$ 仅取得了 $68 \%$ ee, 而缺电 子的配体 L $8 \mathbf{c}$ 以 $89 \%$ 的区域选择性得到了 1,4-加成产物, 产物的 $e e$ 值为 $87 \%$, 将反应体系温度降至 $-5{ }^{\circ} \mathrm{C}$, 产物 的 $e e$ 值提高至 $93 \%$.

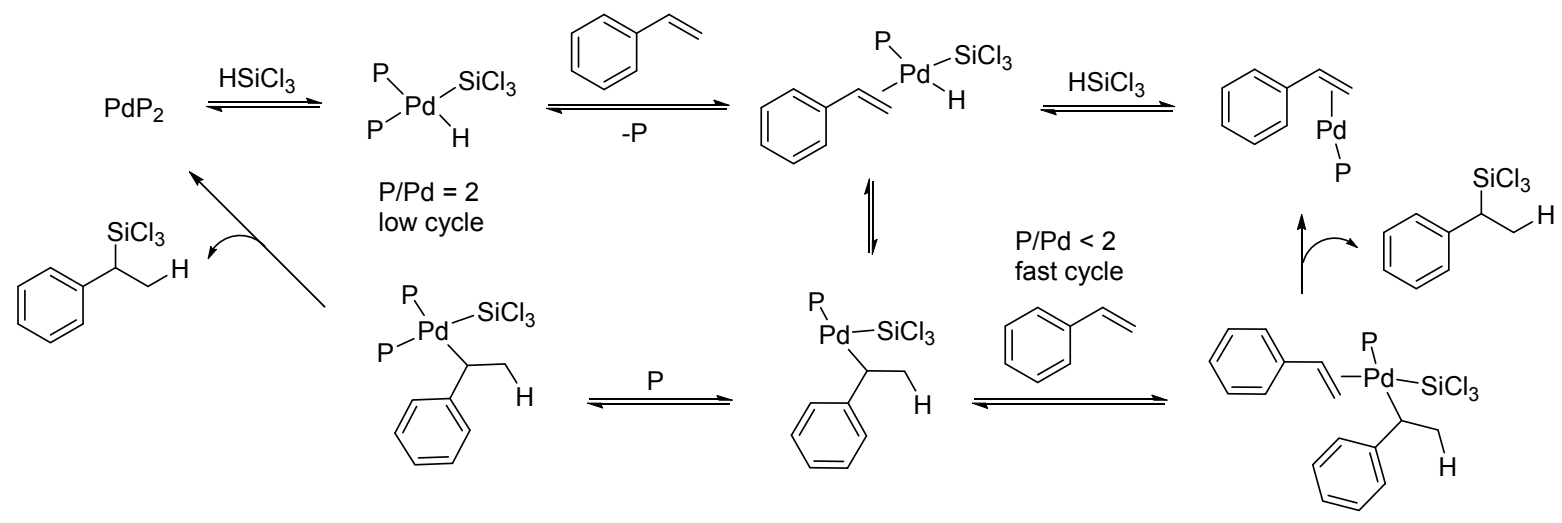

图式 4 基于单膦配体用量的两个催化循环

Scheme 4 Two proposed catalytic cycles depending on loadings of phosphine

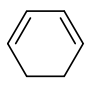

8

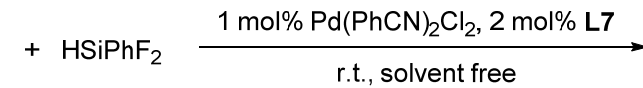

r.t., solvent free

L7a: 20 h, $45 \%$ yield of $9 b, 54 \%$ ee of $12 ;$ L7b: 42 h, $57 \%$ yield of $9 b, 65 \%$ ee of 12 ;

L7c: $\left[\mathrm{Pd}\left(\mathrm{C}_{3} \mathrm{H}_{5}\right) \mathrm{Cl}\right]_{2}$ as precursor, $20 \mathrm{~h}, 58 \%$ yield of $\mathbf{9 b}, 77 \%$ ee of 12

图式 5 平面手性膦配体 $\mathbf{L} 7$ 在环己二烯的不对称硅氢化反应中的应用

Scheme 5 Application of planar chiral phosphines L7 in the asymmetric hydrosilylation of cyclohexadiene

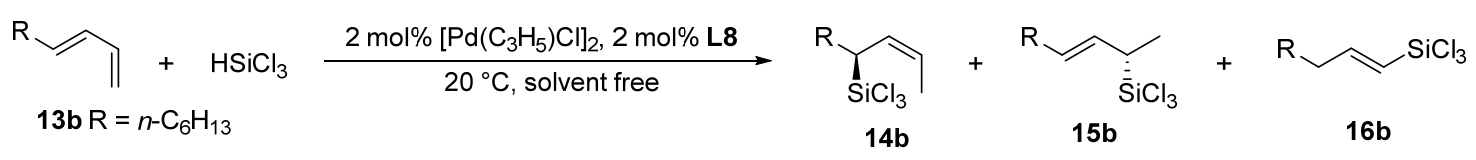

L8a: 29 h, 76\% ee of 14b, 14b : 15b : 16b = $94: 6: 0 ;$ L8b: 96 h, $68 \%$ ee of 14b, 14b : 15b: 16b = $82: 3: 15$

L8c: $25 \mathrm{~h}, 87 \%$ ee of $14 b, 14 b: 15 b: 16 b=87: 10: 3 ;$ L8c: $-5{ }^{\circ} \mathrm{C}, 168 \mathrm{~h}, 93 \%$ ee of $14 b, 14 b: 15 b: 16 b=89: 9: 2$ 
在此基础上, Hayashi 等 ${ }^{[21]}$ 将含二茂铁结构的手性 配体 L7 和 L8 应用于钯催化的 1-丁烯-3-炔(18a)的不对 称硅氢化反应中, 得到了轴手性的丙二烯基硅烷. 他们 发现产物的光学纯度取决于底物 4 位取代基的位阻. 相同条件下, 位阻小的底物, 催化剂选择性较低(表 2 , Entries 1,2). 当底物 4 位为叔丁基(18b)时, 配体 L7a 在 $20{ }^{\circ} \mathrm{C}$ 下, 反应 $9 \mathrm{~h}$, 以 $81 \%$ 的产率得到 $72 \% e e$ 的产物 (表 2, Entry 3). 含两个二茂铁基的配体 L8a 催化活性较 低, 但所得产物 $e e$ 值升为 $85 \%$ (表 2, Entry 4). 进一步将 反应温度降至 $0{ }^{\circ} \mathrm{C}$, ee 值上升至 $90 \%$ (表 2, Entry 5). 2006 年, Hayashi 课题组 ${ }^{[22]}$ 合成了 2 位及 5 位为轴手性 的联二䒺基取代的新型手性磷杂环戊二烯及其金属茂, 并将其一并应用于 $18 \mathrm{~b}$ 的不对称硅氢化反应中. 磷杂二 茂铁配体 $\mathbf{L 9}$ 在 $0{ }^{\circ} \mathrm{C}$ 下反应 $72 \mathrm{~h}$, 得到了 $92 \% e e$ 的产物 21(表 2, Entry 6).

表 2 平面手性膦配体 L7a、L8a 及 $\mathbf{L 9}$ 在钯催化的 1,3-烯炔 的不对称硅氢化反应中的应用 ${ }^{a}$

Table 2 Application of planar chiral phosphines L7a, L8a and $\mathbf{L 9}$ in the asymmetric hydrosilylation of 1,3-enynes

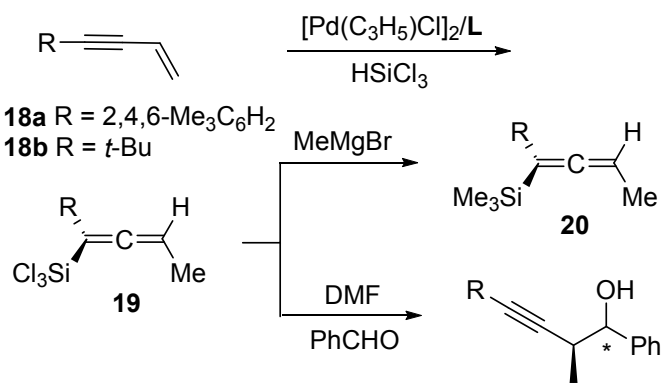

21

\begin{tabular}{lcclcc}
\hline Entry & $\mathbf{1 8}$ & $\mathbf{L}$ & \multicolumn{1}{c}{ Condition } & Yield/\% & $e e / \%$ of $\mathbf{2 0}$ \\
\hline 1 & $\mathbf{1 8 a}$ & $\mathbf{L 7 a}$ & $20{ }^{\circ} \mathrm{C}, 18 \mathrm{~h}$ & 54 & 56 \\
2 & $\mathbf{1 8 a}$ & $\mathbf{L 8 a}$ & $20{ }^{\circ} \mathrm{C}, 64 \mathrm{~h}$ & 90 & 77 \\
3 & $\mathbf{1 8 b}$ & $\mathbf{L 7 a}$ & $20{ }^{\circ} \mathrm{C}, 9 \mathrm{~h}$ & 81 & 72 \\
4 & $\mathbf{1 8 b}$ & $\mathbf{L 8 a}$ & $20{ }^{\circ} \mathrm{C}, 48 \mathrm{~h}$ & 59 & 85 \\
5 & $\mathbf{1 8 b}$ & $\mathbf{L 8 a}$ & $0{ }^{\circ} \mathrm{C}, 160 \mathrm{~h}$ & 37 & 90 \\
$6^{b}$ & $\mathbf{1 8 b}$ & $\mathbf{L 9}$ & $0{ }^{\circ} \mathrm{C}, 72 \mathrm{~h}$ & 82 & 92 \\
\hline
\end{tabular}

${ }^{a}$ All reactions were conducted with $\left[\mathrm{Pd}\left(\mathrm{C}_{3} \mathrm{H}_{5}\right) \mathrm{Cl}\right]_{2}$ as precursor, $1 \mathrm{~mol} \%$ catalyst, [Ligand $] /[\mathrm{Pd}]=2.2$, solvent free unless otherwise stated. ${ }^{b} e e$ of $\mathbf{2 1}$.

\section{2 轴手性单齿膦配体}

轴手性配体在整个不对称领域占据着重要地位, 如 具有联二荎骨架的轴手性双膦配体 BINAP, 因成功应用 于多种类型的不对称催化反应而被称为 “明星分子” [23]. 然而, Hayashi 等 ${ }^{[24]}$ 发现金属钯与 BINAP 形成的络合物 在高达 $60{ }^{\circ} \mathrm{C}$ 时, 仍然不能使烯烃发生不对称硅氢化反 应. 这可能是双膦配体与钯配位后, 形成了配位稳定的 络合物, 占据了钯的两个空轨道, 底物不能与金属有效 配位, 故催化反应不能顺利进行. 而单膦配体就能够以
一个配体与钯配位, 进而催化不对称反应. 轴手性的单 齿膦配体(图 3)底物普适性好, 在烷基烯烃、苯乙烯、1,3二烯烃等底物中均取得了优异的催化效果.

Hayashi 等合成了联二荎骨架的配体 L10, 并将其 应用于一系列链状末端烯烃的不对称硅氢化反应中，首 次以高区域选择性、高收率及优异的对映选择性生成手 性仲醇(表 3). 如采用 $\left[\mathrm{Pd}\left(\mathrm{C}_{3} \mathrm{H}_{5}\right) \mathrm{Cl}\right]_{2}$ 作为催化剂前体, 当 催化剂的用量为 $0.1 \mathrm{~mol} \%$ 时, $40{ }^{\circ} \mathrm{C}$ 下反应 $24 \mathrm{~h}$, 配体 L10a (MeO-MOP) 在 1-辛烯(22a)与三氯硅烷的不对称硅 氢化反应中, 取得了 $83 \%$ 的产率, 直链产物和支链产 物的比例为 7：93, 支链产物的对映选择性为 $95 \%$ (表 3, Entry 1), 当甲氧基被异丙氧基取代时得到配体 L10b，其对应产物的ee值略有下降至 91\% (表 3, Entry 2); 甲氧基被更大位阻的茮氧取代配体 L10c 虽然取 得了高达 $95 \% e e$, 可是区域选择性下降明显, 仅为 $80: 20$ (表 3, Entry 3). 将 $\mathrm{MeO}-\mathrm{MOP}$ 中的甲氧基变成乙 基时所得的配体 L10d，无论是区域选择性还是立体选 择性均与 $\mathrm{MeO}-\mathrm{MOP}$ 相近, 说明氧原子并不是获取高选 择性的必要条件(表 3, Entry 4).

表 3 轴手性单膦配体 $\mathbf{L 1 0}$ 在链状端烯的不对称硅氢化反应 中的应用 ${ }^{a}$

Table 3 Application of axially chiral phosphines $\mathbf{L 1 0}$ in the asymmetric hydrosilylation of acyclic terminated alkenes

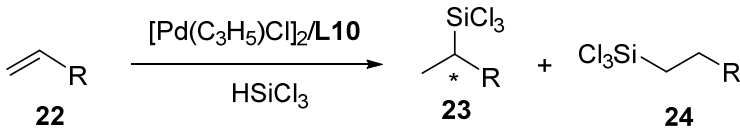

22a $\mathrm{R}=n-\mathrm{C}_{6} \mathrm{H}_{13}, 22 \mathrm{~b} \mathrm{R}=n-\mathrm{C}_{4} \mathrm{H}_{9}, 22 \mathrm{c} \mathrm{R}=n-\mathrm{C}_{10} \mathrm{H}_{21}$,

22d R $=\mathrm{PhCH}_{2} \mathrm{CH}_{2}$, 22e $\mathrm{R}=\mathrm{PhCH}_{2} \mathrm{CH}_{2} \mathrm{CH}_{2}, 22 \mathrm{f}=c-\mathrm{C}_{6} \mathrm{H}_{11}$

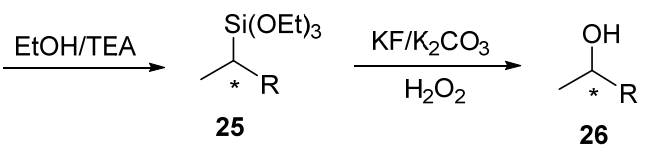

\begin{tabular}{|c|c|c|c|c|c|c|}
\hline Entry & 22 & $\mathbf{L}$ & Condition & Yield/\% & $23: 24$ & $\%$ of 26 \\
\hline 1 & $22 a$ & L10a & $40{ }^{\circ} \mathrm{C}, 24 \mathrm{~h}$ & 83 & $93: 7$ & 95 \\
\hline 2 & $22 a$ & L10b & $40{ }^{\circ} \mathrm{C}, 24 \mathrm{~h}$ & 88 & $90: 10$ & 91 \\
\hline 3 & $22 a$ & L10c & $40{ }^{\circ} \mathrm{C}, 24 \mathrm{~h}$ & 85 & $80: 20$ & 95 \\
\hline 4 & $22 a$ & L10d & $40{ }^{\circ} \mathrm{C}, 24 \mathrm{~h}$ & 80 & $90: 10$ & 93 \\
\hline 5 & $22 b$ & L10a & $40{ }^{\circ} \mathrm{C}, 24 \mathrm{~h}$ & 91 & $89: 11$ & 94 \\
\hline 6 & $22 c$ & L10a & $40{ }^{\circ} \mathrm{C}, 72 \mathrm{~h}$ & 90 & $94: 6$ & 95 \\
\hline 7 & 22d & L10a & $40{ }^{\circ} \mathrm{C}, 24 \mathrm{~h}$ & 90 & $81: 19$ & 97 \\
\hline $8^{b}$ & $22 \mathrm{e}$ & L10a & $30{ }^{\circ} \mathrm{C}, 60 \mathrm{~h}$ & 81 & $80: 20$ & 92 \\
\hline 9 & $22 f$ & L10a & $40{ }^{\circ} \mathrm{C}, 24 \mathrm{~h}$ & 100 & $66: 34$ & 96 \\
\hline
\end{tabular}

${ }^{a}$ All reactions were conducted with $\left[\mathrm{Pd}\left(\mathrm{C}_{3} \mathrm{H}_{5}\right) \mathrm{Cl}_{2}\right.$ as precursor, $0.1 \mathrm{~mol} \%$ catalyst, $[\mathbf{L}] /[\mathrm{Pd}]=2$, solvent free unless otherwise stated. ${ }^{b} \mathrm{THF}$ as solvent.

$\mathrm{MeO}-\mathrm{MOP}$ 不仅在链状端烯的不对称硅氢化反应中 取得了优异的催化效果, 在环状烯烃中也有较好的应用 (Scheme 6). 1992 年, Hayashi 等 ${ }^{[25]}$ 将 Pd/MeO-MOP 催化 剂应用于降冰片烯和三氯硅烷的不对称硅氢化反应中, 在催化剂的用量为 $0.01 \mathrm{~mol} \%$ 时， $-20{ }^{\circ} \mathrm{C}$ 下反应 $3 \mathrm{~d}$, 
<smiles>[R]c1ccc2ccccc2c1-c1c(-c2ccccc2)ccc2ccccc12</smiles>

L10<smiles>COc1ccc2ccc3ccccc3c2c1-c1c(-c2ccccc2)ccc2ccc3ccccc3c12</smiles>

L14
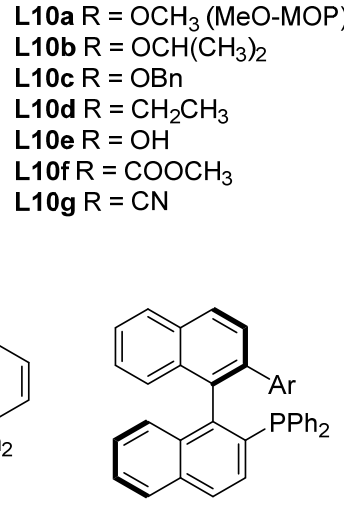

L15

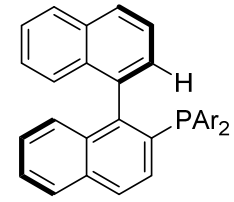

L11a $\mathrm{Ar}=\mathrm{Ph}(\mathrm{H}-\mathrm{MOP})$ L11b $\mathrm{Ar}=3,5-\left(\mathrm{CF}_{3}\right)_{2} \mathrm{C}_{6} \mathrm{H}_{3}$

$\mathrm{L} 15 \mathrm{a} \mathrm{Ar}=\mathrm{Ph}$

L15b Ar $=4-\mathrm{CH}_{3} \mathrm{OC}_{6} \mathrm{H}_{4}$ L15c Ar $=4-\mathrm{CF}_{3} \mathrm{C}_{6} \mathrm{H}_{4}$ L15d $\mathrm{Ar}=3-\mathrm{CH}_{3} \mathrm{C}_{6} \mathrm{H}_{4}$ L15e $\mathrm{Ar}=3,5-\left(\mathrm{CH}_{3}\right)_{2} \mathrm{C}_{6} \mathrm{H}_{3}$ L15f $\mathrm{Ar}=3,5-\left(\mathrm{CH}_{3}\right)_{2}-4-\mathrm{CH}_{3} \mathrm{OC}_{6} \mathrm{H}_{2}$<smiles>C1=CC2C=CC=C(c3c(-c4ccccc4)cccc3-c3ccccc3)C2C=C1</smiles>

L12<smiles>CC1=C(c2c(C)cc(C)cc2Pc2ccccc2)C2C=CC=CC2C=C1</smiles>

L13<smiles>[R]c1ccc2cc(-c3c(-c4ccccc4)ccc4ccc([R])cc34)ccc2c1</smiles>

L16 R $=n-\mathrm{C}_{8} \mathrm{H}_{17} ; \mathrm{Ar}=3,5-\left(\mathrm{CH}_{3}\right)_{2}-4-\mathrm{CH}_{3} \mathrm{OC}_{6} \mathrm{H}_{2}$<smiles>[R2]c1ccc2ccccc2c1-c1c([R20])ccc2ccccc12</smiles>

L17

L17a R $=\mathrm{H}, \mathrm{Ar}=3,5-(t-\mathrm{Bu})_{2} \mathrm{C}_{6} \mathrm{H}_{3}$ L17b R $=\mathrm{CH}_{3} \mathrm{O}, \mathrm{Ar}=3,5-(t-\mathrm{Bu})_{2} \mathrm{C}_{6} \mathrm{H}_{3}$ L17c $\mathrm{R}=\mathrm{CN}, \mathrm{Ar}=3,5-(t-\mathrm{Bu})_{2} \mathrm{C}_{6} \mathrm{H}_{3}$

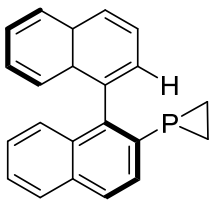

L20

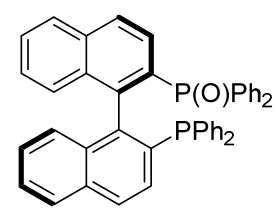

L18

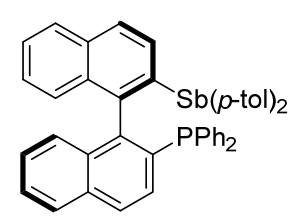

L19

图 3 用于烯烃不对称硅氢化反应的轴手性单齿膦配体

Figure 3 Axially chiral monodentate phosphines used in the asymmetric hydrosilylation of alkenes

以定量的产率生成外-2-三氯硅基降冰片烷, 经氧化后 得到 $e e$ 值为 $96 \%$ 的手性外-降冰片醇. 降冰片烯的环上 的酯基在硅氢化反应中不受影响. 此外, 改变三氯硅氢 用量, 降冰片二烯可选择性地生成单硅氢化产物或双硅 氢化产物. 若三氯硅氢的用量为 1 equiv. 时, 生成单硅氢 化产物, ee 值为 $95 \%$; 若三氯硅氢的用量为 2.5 equiv. 时, 两个双键均发生反应, 所得产物的 $e e$ 值大于 $99 \%$. 随 后, 他们将 $\mathrm{Pd} / \mathrm{MeO}-\mathrm{MOP}$ 应用范围进一步拓展到呋喃 类底物的不对称硅氢化反应中 ${ }^{[26]}$. 2,3-二氢呋喃在催化 剂的用量为 $0.2 \mathrm{~mol} \%$ 时, $25{ }^{\circ} \mathrm{C}$ 下反应 $12 \mathrm{~h}$, 以 $86 \%$ 的 产率生成 2-三氯硅基四氢呋喃, 与格氏试剂作用后, 得 到 $e e$ 值为 $82 \%$ 的手性 2-三甲基硅基四氢呋喃. 2,5-二氢 呋喃在催化剂的用量为 $0.1 \mathrm{~mol} \%$ 时, $40{ }^{\circ} \mathrm{C}$ 下反应 $24 \mathrm{~h}$, 以 $65 \%$ 的产率生成 3 -三氯硅基四氢呋喃, 经氧化后, 以 $83 \%$ 的收率得到 $e e$ 值为 $95 \%$ 的手性醇. 他们进一步 将底物拓展到酯基取代的 7-氧杂二环 $[2,2,1]$ 庚烷中, 以 $100 \%$ 的区域选择性生成了 $95 \%$ ee 的含多个手性中心产 物.

配体 MeO-MOP 虽然在脂肪烯烃的硅氢化反应中取
得了优异的催化性能, 但在苯乙烯的不对称硅氢化反应 中, 在 $0{ }^{\circ} \mathrm{C}$ 及无溶剂的条件下, 仅取得了 $14 \% e e^{[27]}$. 采 用苯作为溶剂，催化剂的选择性升高至 $71 \%$ 。然而，令 人遗憾的是, 其他 $2^{\prime}$ 位为给电子基的配体 $(\mathbf{L 1 0 d} 、 \mathbf{L 1 0 e})$ 或吸电子基的配体 $(\mathbf{L 1 0 f} 、 \mathbf{L 1 0 g})$ 取得的 $e e$ 值均很低, 说 明 2 位电子效应对于催化剂选择性并不起决定作用. 后 来, 他们发现配体中 $2^{\prime}$ 位的位阻对于选择性有很大影响, 2'位为氢的配体 L11a (H-MOP)取得了高达 95\% ee 值. 2001 年, Hayashi 等 ${ }^{[28]}$ 合成了结构与 H-MOP 类似的 2 位 磷原子上为吸电子基 $\left[3,5-\left(\mathrm{CF}_{2}\right)_{2} \mathrm{C}_{6} \mathrm{H}_{3}\right]$ 的配体 $\mathbf{L 1 1 b}$, 其 在钯催化的苯乙烯与三氯硅氢的不对称催化反应中, 取 得了优异的催化效果. 在 $0{ }^{\circ} \mathrm{C}$ 下, 当催化剂的用量为 $0.1 \mathrm{~mol} \%$ 时, 底物在 $1 \mathrm{~h}$ 内完全转化, 所得产物的 $e e$ 值 为 $97 \%$. 降低温度至 $-20{ }^{\circ} \mathrm{C}, e e$ 值进一步提高至 $98 \%$. 1995 年, 他们 ${ }^{[29]}$ 通过钯催化的不对称交叉偶联反应合 成了 $2^{\prime}$-位为氢原子的轴手性联芳基骨架的单齿膦配体 L12. 采用 $\left[\mathrm{Pd}\left(\mathrm{C}_{3} \mathrm{H}_{5}\right) \mathrm{Cl}\right]_{2} / \mathbf{L 1 2}$ 作为催化剂, $0{ }^{\circ} \mathrm{C}$ 下反应 $24 \mathrm{~h}$, 以 $68 \%$ 的收率得到了产物, 对映选择性为 $91 \%$. Bringmann 等 ${ }^{[30]}$ 报道了相同骨架的类似配体 L13, 配体. 
$\left.\mathrm{Pd}\left(\mathrm{C}_{3} \mathrm{H}_{5}\right) \mathrm{Cl}\right]_{2} / \mathrm{MeO}-\mathrm{MOP}($ L10a) as catalyst, $[$ L10a]/[Pd] = 2

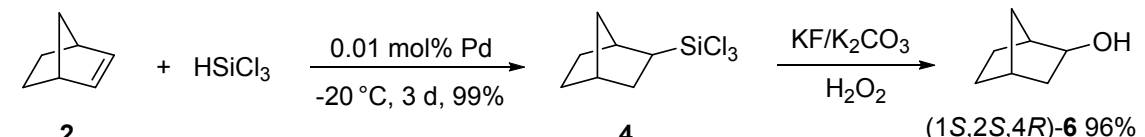

2

$(1 S, 2 S, 4 R)-6$ 96\% ee

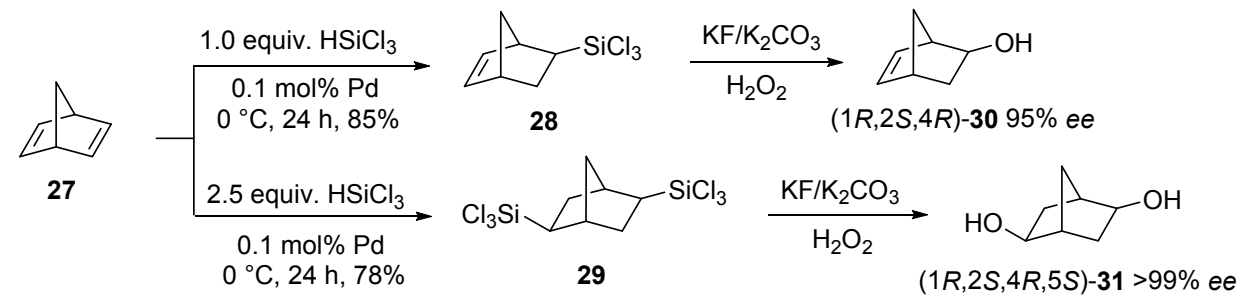

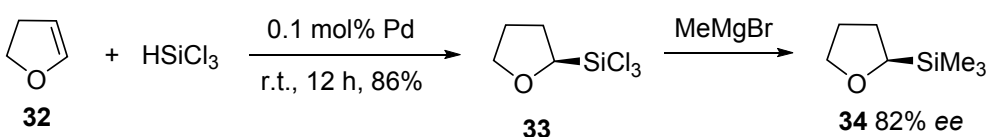

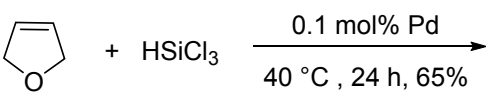

35<smiles>[SiH3]C1CCOC1</smiles><smiles>O=C(O)CC(=O)O[Na]</smiles><smiles>OC1CCOC1</smiles>

37 95\% ee

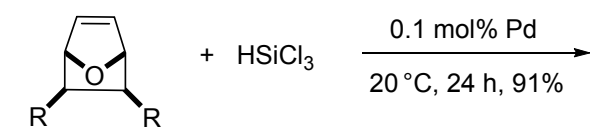

$38 \mathrm{R}=\mathrm{COOCH}_{3}$

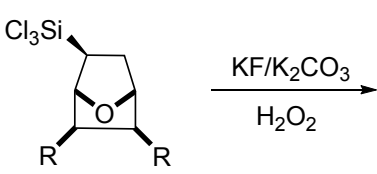

39

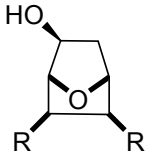

40 95\% ee

图式 $6 \mathrm{MeO}-\mathrm{MOP}$ 在环状烯烃的不对称硅氢化反应中的应用

Scheme 6 Application of MeO-MOP in the asymmetric hydrosilylation of cyclic alkenes

$\mathbf{L 1 3}$ 的 2'为甲基, 在相同条件下仅取得了 $50 \% e e$. 上述 结果表明, $2^{\prime}$ 位取代基在芳基烯烃的不对称硅氢化反应 中扮演着重要角色, 尽管具体的原因还不明了. 其中, 2' 位为氢的配体有着较好的选择性.

1996 年, Hayashi 等 ${ }^{[31]}$ 首次将 MOP 类配体应用于 $\left[\mathrm{Pd}\left(\mathrm{C}_{3} \mathrm{H}_{5}\right) \mathrm{Cl}\right]_{2}$ 催化的环状 1,3 -二烯烃与三氯硅烷的反 应中. 当底物为环戊二烯时, $\mathrm{MeO}-\mathrm{MOP}$ 在 $20{ }^{\circ} \mathrm{C}$ 下反应 $14 \mathrm{~h}$, 底物完全转化, 所得产物的 ee 值为 $39 \%$ (表 4 , Entry 1). 同样条件下, H-MOP 的选择性仅为 $28 \%$ (表 4, Entry 2). 与 $\mathrm{MeO}-\mathrm{MOP}$ 结构类似的联二菲骨架的配体 L14 虽然活性不高, 却取得了 $80 \%$ 的 $e e$ 值(表 4, Entry 3). 催化活性不高的原因可能是 Pd-Ar-MOP 催化体系在 双烯与三氯氢硅烷加成反应体系中的溶解性较低引起 的. 后来, 他们 ${ }^{[32]}$ 将 $2^{\prime}$ 位为芳基的配体 $\mathbf{L 1 5}$ 应用于环龙 二烯的不对称硅氢化反应中. 研究结果表明, $0{ }^{\circ} \mathrm{C}$ 下, 钯催化剂的用量为 $0.25 \mathrm{~mol} \%$ 时, 2 '位芳基越富有电子, 位阻越大, 所生成的产物 $e e$ 值越高. 如配体 L15a 的 $e e$ 值为 69\%(表 4, Entry 4), 富电子配体 L15b 和 L15e 的 $e e$ 值分别为 76\%(表 4, Entry 5)和 79\%(表 4, Entry 8), 而既
富有电子又具有较大位阻的配体 L15f, ee 值高达 $88 \%$ (表 4, Entry 9). 进一步降低反应温度至 $-20{ }^{\circ} \mathrm{C}$, ee 值上升至 $90 \%$ (表 4, Entry 10). 与之相对应的是缺电子 基的配体 L15c 在相同条件下 $\left(0{ }^{\circ} \mathrm{C}\right)$ 仅取得了 $47 \%$ ee (表 4, Entry 6). 而当底物为环已二烯时, $0{ }^{\circ} \mathrm{C}$ 下, 配体 L15f 仅取得 79\% ee(表 4, Entry 13). 进一步降低反应温度不 能生成目标产物, 这可能是钯催化剂低温下不能溶解于 反应体系所致. 为了提高此催化体系的溶解性, 他们 ${ }^{[33]}$ 在配体 L15f 的 6 位和 6'位上引入长链烷基, 得到配体 L16. 配体 L16 能很好的溶于反应体系, 将其应用于钯 催化的环状 1,3-二烯烃与三氯硅烷的加成反应中. 当底 物为环戊二烯, 钯催化剂用量为 $0.25 \mathrm{~mol} \%$, 配体为 0.5 mol $\%$ 时， $-30{ }^{\circ} \mathrm{C}$ 下，配体 $\mathbf{L 1 6}$ 取得了 $75 \%$ 的产率以及 $91 \%$ 的 $e e$ 值(表 4, Entry 11). $-10{ }^{\circ} \mathrm{C}$ 下, 配体 L16 在 1,3-环已二烯的不对称催化中, 取得了 $70 \%$ 的产率以及 $83 \%$ 的 $e e$ 值(表 4, Entry 12). 除此之外, 轴手性的单齿膦 配体还能催化链状 1,3-二烯烃的硅氢加成反应, 然而仅 取得了中等对映选择性 ${ }^{[34]}$. 
表 4 轴手性膦配体用于的环状 1,3-二烯烃的不对称硅氢化反 应 $^{a}$

Table 4 Axially phosphines used in the asymmetric hydrosilylation of cyclic 1,3-dienes
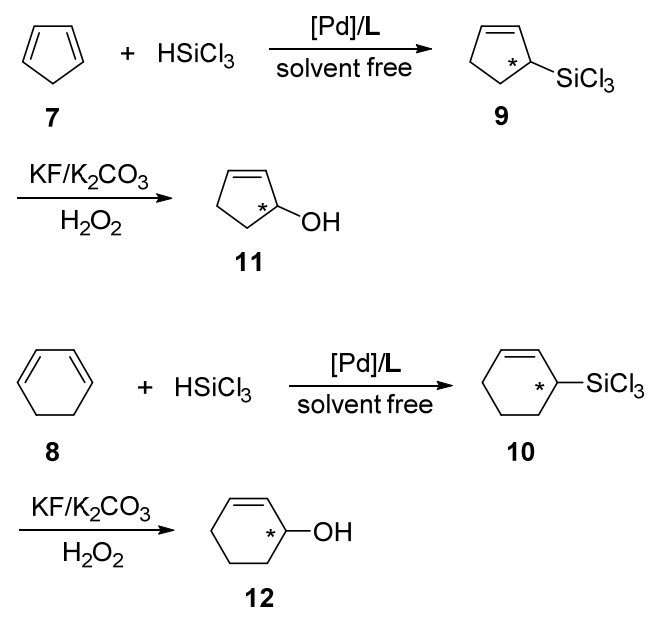

\begin{tabular}{|c|c|c|c|c|c|}
\hline Entry & $7 / 8$ & $\mathbf{L}$ & Condition & $\begin{array}{c}\text { Yield } / \% \text { of } \\
\mathbf{9 / 1 0} \\
\end{array}$ & $\begin{array}{c}e e / \% \text { of } \\
\mathbf{1 1 / 1 2}\end{array}$ \\
\hline $1^{b}$ & 7 & L10a & $20{ }^{\circ} \mathrm{C}, 14 \mathrm{~h}$ & 100 & 39 \\
\hline $2^{b}$ & 7 & L11a & $20{ }^{\circ} \mathrm{C}, 3 \mathrm{~h}$ & 91 & 28 \\
\hline $3^{b}$ & 7 & L14 & $20{ }^{\circ} \mathrm{C}, 120 \mathrm{~h}$ & 99 & 80 \\
\hline 4 & 7 & L15a & $0{ }^{\circ} \mathrm{C}, 24 \mathrm{~h}$ & 84 & 69 \\
\hline 5 & 7 & L15b & $0{ }^{\circ} \mathrm{C}, 24 \mathrm{~h}$ & 84 & 76 \\
\hline 6 & 7 & L15c & $0{ }^{\circ} \mathrm{C}, 24 \mathrm{~h}$ & 83 & 47 \\
\hline 7 & 7 & L15d & $0{ }^{\circ} \mathrm{C}, 24 \mathrm{~h}$ & 85 & 76 \\
\hline 8 & 7 & L15e & $0{ }^{\circ} \mathrm{C}, 24 \mathrm{~h}$ & 83 & 79 \\
\hline 9 & 7 & L15f & $0{ }^{\circ} \mathrm{C}, 24 \mathrm{~h}$ & 79 & 88 \\
\hline 10 & 7 & L15f & $-20{ }^{\circ} \mathrm{C}, 72 \mathrm{~h}$ & 89 & 90 \\
\hline 11 & 7 & L16 & $-30{ }^{\circ} \mathrm{C}, 168 \mathrm{~h}$ & 75 & 91 \\
\hline 12 & 8 & L16 & $-10{ }^{\circ} \mathrm{C}, 168 \mathrm{~h}$ & 70 & 83 \\
\hline 13 & 8 & L15f & $0{ }^{\circ} \mathrm{C}, 72 \mathrm{~h}$ & 75 & 79 \\
\hline
\end{tabular}

${ }^{a}$ All reactions were conducted with $\left[\mathrm{Pd}\left(\mathrm{C}_{3} \mathrm{H}_{5}\right) \mathrm{Cl}\right]_{2}$ as precursor, $0.25 \mathrm{~mol} \%$ catalyst, $[\mathrm{L}] /[\mathrm{Pd}]=2$, solvent free unless otherwise stated. ${ }^{b} 0.1 \mathrm{~mol} \%$ catalyst.

2004 年, Pregosin 等 ${ }^{[35]}$ 合成并表征了配体 L17. 与 典型的 MOP 类配体相比, 这类配体结构的显著特点是 联二菜骨架的 2 位磷原子上连有大位阻的芳基. 他们将 这些配体与 Hayashi 之前报道的 2 位为二苯基膦的配体 一并应用于苯乙烯的不对称硅氢化反应中. 在 $5{ }^{\circ} \mathrm{C}$ 下, 采用 $\left[\mathrm{Pd}\left(\mathrm{C}_{3} \mathrm{H}_{5}\right) \mathrm{Cl}\right]_{2}$ 作为催化剂前体, 三氯硅氢作为硅 烷，当催化剂的用量为 $0.096 \mathrm{~mol} \%$ 时, $2^{\prime}$ 位为氢的配体 L11a (H-MOP) 取得了 92\% ee(表 5, Entry 1), 而在相同 条件下, 较大位阻的配体 L15a 也取得了 $88 \%$ ee(表 5, Entry 2). 当 $2^{\prime}$ 位为甲氧基的配体 $\mathbf{L 1 0 a}(\mathrm{MeO}-\mathrm{MOP})$ 的 $e e$ 值仅为 7\%(表 5, Entry 3), 而与之相对应的大位阻配体 L17b 却取得了 $57 \% e e$ (表 5, Entry 4); 当 2'位为氰基时, 也是大位阻配体的 $e e$ 值远远高于相应的二苯基膦配体 (表 5, Entries 5,vs.6). 他们的研究结果表明, 2 位的位阻 对于催化剂的选择性有着显著的影响，大位阻基团的引 入可能有利于获取高对映选择性的产物. 虽然位阻效应
导致催化剂选择性差异的具体原因还不清楚, 但是, 他 们认为, 大位阻基团使得磷一碳键的旋转受阻，有利于 创造刚性的 “手性口袋”，从而促进催化剂选择性的提 高 $^{[36]}$.

表 5 轴手性膦配体 $\mathbf{L 1 7} \sim \mathbf{L 2 2}$ 在苯乙烯的不对称硅氢化反应 中的应用 ${ }^{a}$

Table 5 Application of axially chiral phosphines $\mathbf{L 1 7} \sim \mathbf{L 2 2}$ in the asymmetric hydrosilylation of styrene

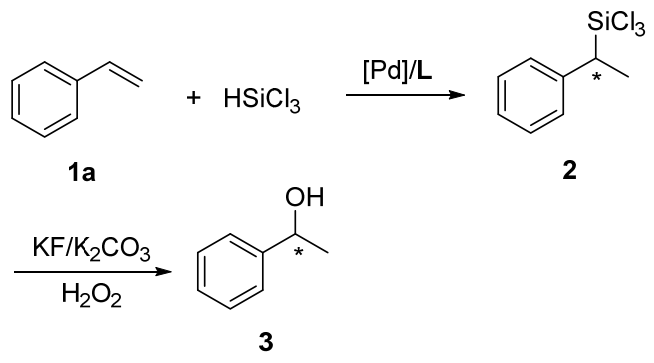

\begin{tabular}{|c|c|c|c|c|}
\hline Entry & $\mathbf{L}$ & Condition & Yield/\% of 2 & $e e / \%$ of $\mathbf{3}$ \\
\hline 1 & L11a & $0{ }^{\circ} \mathrm{C}, 20 \mathrm{~h}$ & 100 & 92 \\
\hline 2 & L17a & $5{ }^{\circ} \mathrm{C}, 16 \mathrm{~h}$ & 100 & 88 \\
\hline 3 & L10a & $5{ }^{\circ} \mathrm{C}, 16 \mathrm{~h}$ & 100 & 7 \\
\hline 4 & L17b & $0{ }^{\circ} \mathrm{C}, 43 \mathrm{~h}$ & 100 & 57 \\
\hline 5 & L10g & $5{ }^{\circ} \mathrm{C}, 2 \mathrm{~h}$ & 100 & 42 \\
\hline 6 & L17c & $5{ }^{\circ} \mathrm{C}, 16 \mathrm{~h}$ & 100 & 81 \\
\hline 7 & L18 & r.t., $70 \mathrm{~h}$ & 100 & 18 \\
\hline $8^{b}$ & L18 & r.t., $70 \mathrm{~h}$ & 100 & 72 \\
\hline 9 & L19 & $0{ }^{\circ} \mathrm{C}, 10 \mathrm{~h}$ & 78 & 95 \\
\hline 10 & L20 & r.t., $24 \mathrm{~h}$ & $>99$ & 80 \\
\hline $11^{b}$ & L21 & r.t., $18 \mathrm{~h}$ & 92 & 91 \\
\hline $12^{c}$ & L22a & r.t., $3 \mathrm{~h}$ & 63 & 54 \\
\hline $13^{c}$ & L22b & r.t., $3 \mathrm{~h}$ & 90 & 54 \\
\hline
\end{tabular}

${ }^{a}$ All reactions were conducted with $\left[\mathrm{Pd}\left(\mathrm{C}_{3} \mathrm{H}_{5}\right) \mathrm{Cl}\right]_{2}$ as precursor, $0.1 \mathrm{~mol} \%$ catalyst, $[\mathbf{L}] /[\mathrm{Pd}]=2$, solvent free unless otherwise stated. ${ }^{b}$ Benzene as solvent. ${ }^{c} 2.5 \mathrm{~mol} \%$ catalyst.

此外, Gladiali 等 ${ }^{[37]}$ 将双膦配体 BINAP 部分氧化得 到单齿膦配体 L18 (BINAPO), 并将其应用于苯乙烯的 不对称硅氢化反应中. 室温下，当配体与金属的比例为 2 时, 底物与三氯硅氢反应 $70 \mathrm{~h}$ 后, 底物完全转化, 仅 取得了 $18 \% e e($ 表 5 , Entry 7). 当采用苯作为溶剂时, 催 化剂的对映选择性升高至 72\%(表 5, Entry 8). 受此启发, 2004 年 Kurita 等 ${ }^{[38]}$ 报道了单膦配体 L19 (BINAPSb)的合 成及性能研究. 他们将其应用于苯乙烯的不对称硅氢化 反应中, $0{ }^{\circ} \mathrm{C}$ 下应 $10 \mathrm{~h}$ 时, 以 $78 \%$ 的收率、 $95 \%$ 的 $e e$ 得到 $S$-苯乙醇(表 5, Entry 9). 而双铋配体在相同条件下 反应 $24 \mathrm{~h}$, 仅取得了 $12 \%$ ee . 这一结果说明, 配体中单 个磷原子的存在是该反应中获得较好催化效果的必要 条件.

2011 年, Higham 等 ${ }^{[39]}$ 采用联二萘骨架的伯膦和二 氯乙烷作为原料，在甲基锂的作用下，合成了稳定的联 二䒺骨架的手性磷杂环丙烷 L20. 将 2 equiv.配体与顺 
式 $[\mathrm{Pd}(\mathrm{COD}) \mathrm{Cl}]_{2}$ 反应，通过高分辨质谱、核磁共振及 $\mathrm{X}$ 射线单晶衍射等研究了手性磷杂环丙烷与后过渡金属 的配位行为, 了解到具有对热及空气稳定的手性磷杂环 丙烷与后过渡金属配位时稳定性并没有下降. 他们将这 种新型的稳定的手性磷杂环丙烷配体用于 $\left[\mathrm{Pd}\left(\mathrm{C}_{3} \mathrm{H}_{5}\right) \mathrm{Cl}\right]_{2}$ 催化的苯乙烯的不对称硅氢化反应中, 在反应条件未经优化的前提下，取得了 $80 \% \mathrm{ee}$ (表 5 , Entry 10).

2012 年, Lemaire 等 ${ }^{[40]}$ 从手性 BINOL 出发经过 5 步反应得到了兼具 P 手性和轴手性的配体 L21. 室温下, 采用 $\left[\mathrm{Pd}\left(\mathrm{C}_{3} \mathrm{H}_{5}\right) \mathrm{Cl}\right]_{2}$ 作为催化剂前体, 当催化剂的用量 为 $0.04 \mathrm{~mol} \%$ 时, 配体取得了 $91 \%$ 的对映选择性(表 5 , Entry 11). 同时, 他们发现仅改变配体中磷的构型, 并 不影响产物的构型; 而改变轴手性的构型, 产物的构型 发生变化. 这说明磷原子的构型在不对称诱导中不起决 定性的作用, 配体本身的位阻/电子效应可能更大程度 上影响催化性能. 最近, Panossian 等 ${ }^{[41]}$ 合成了轴手性的 “Buchwald 型” 联苯基单齿膦配体 $\mathbf{L 2 2}$, 并将其应用于 苯乙烯的不对称硅氢化反应中, 虽然催化剂在该反应中 活性较好，然而仅取得了 54\% ee(表 5, Entries 12,13).

\section{3 其他手性单齿膦配体}

1972 年, Kumada 等 ${ }^{[6,42]}$ 将萜衍生薄荷基二苯基膦 L23 和新薄荷基二苯基膦 L24(图 4)应用于苯乙烯的不 对称硅氢化反应中. 室温下, 当采用 $\mathrm{Pd}(\mathrm{PhCN})_{2} \mathrm{Cl}_{2}$ 作为 催化剂前体, 室温下反应 $5 \mathrm{~h}$, 配体 L23 取得了 $34 \% e e$, 配体 L24 取得了 $22 \%$ ee. 而磷手性的配体 L25 需要高达 $120{ }^{\circ} \mathrm{C}$ 的反应温度, 仅得到了 $2 \%$ ee. 这些配体活性差 异可能在于配体 L25 位阻较小, 使得有 2 equiv. 的配体 与金属配位, 底物插入时需要更大能量使得 1 equiv.配 体配位解离. 手性三烷基膦配体 $\mathbf{L 2 6}^{[43]}$ 应用于环戊二烯 的硅氢化反应中, 当配体与金属的比例为 1 时, 25 $30{ }^{\circ} \mathrm{C}$ 下，取得了 $70 \%$ 的产率以及 $54 \% e e$; 而配体与金 属比例为 2 时, 在高达 $70{ }^{\circ} \mathrm{C}$ 温度下, 仅取得了 $26 \%$ 的 产率以及 $44 \%$ ee. 第二当量的富电子三烷基膦配体在 该反应中很明显是不利的. Achiwa 等 ${ }^{[44]}$ 将 $S$-坃氨醇衍生 的手性 $\beta$-胺基烷基磷配体 $\mathbf{L 2 7}$ 在环戊二烯的不对称硅 氢化反应中选择性非常低. 在相同条件下，配体 L28a 和 L28c 取得的对映选择性分别为 $61 \%$ 和 $72 \%$. 而配体 $\mathbf{L 2 8 b}$ 仅取得了 7\% ee, 说明 $\beta$-胺基上的氢原子对于选择 性是十分重要的.

近年来, 人工合成的螺旋聚合物的应用为不对称催 化中构筑手性环境提供了新方法. 2010 年, Suginome 等 ${ }^{[45]}$ 采用手性有机钯引发剂, 通过不对称活性聚合反 应合成了结构新颖的含二苯基膦单元的聚喹喔啉结构 的手性聚合物配体 L29. 他们将螺旋手性聚合物作为配

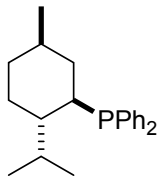

L23

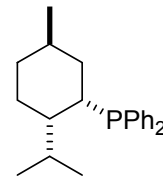

L24

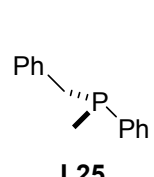

L25

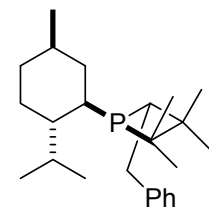

L26

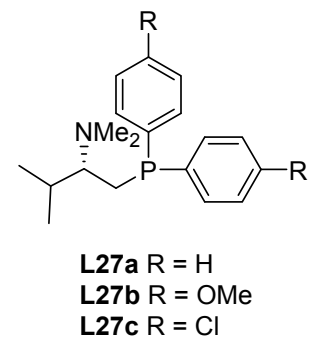

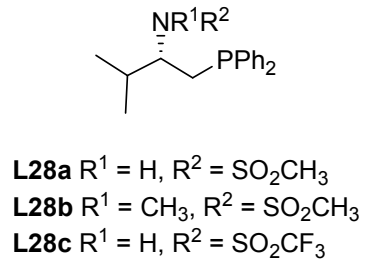

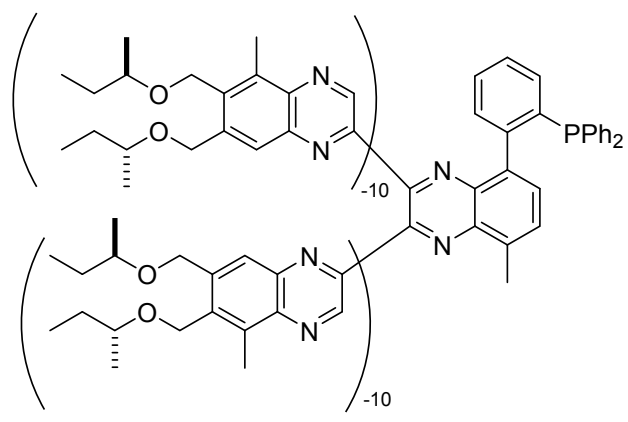

L29

图 4 用于烯烃不对称硅氢化反应的其他手性单齿膦配体 Figure 4 Structures of other chiral monodentate phosphines used in the asymmetric hydrosilylation of alkenes

体，应用于钯催化的苯乙烯的不对称硅氢化反应中. 其 中, 右手螺旋的聚合物配体得到了光学纯度为 $96 \%$ 的苯 乙醇. 值得一提的是，配体与金属形成的络合物不溶于 反应体系中, 便于催化剂的回收与再利用. 催化剂回收 9 次后, 其活性和选择性基本保持不变. 另外, 溶剂可 控制聚合物骨架的构型，因此可以通过一种手性催化剂 可控生成两种不同构型的产物.

\section{2 手性单齿亚磷酰胺酯配体在钯催化的烯烃的 不对称硅氢化反应中的应用}

亚磷酰胺酯配体结构可分为亚磷酰基和胺基两部 分. 早在 1994 年 Feringa 等 ${ }^{[46]}$ 首次报道了联二菜骨架的 单齿手性亚磷酰胺酯配体的合成，在随后的几年，将其 成功地运用于 1,4 -共轭加成 ${ }^{[47]}$ 、不对称氢化反应 ${ }^{[8]}$ 以及 烯丙基取代反应 ${ }^{\left[{ }^{[9]}\right.}$ 中，取得了高活性及高对映选择性. 相对而言, 亚磷酰胺配体在烯烃硅氢化反应中的报道较 少. 目前. 亚磷酰胺酯配体(图 5)主要应用于苯乙烯类底 物的不对称硅氢化反应中.

2002 年, Johannsen 等 ${ }^{[50]}$ 首次将 Feringa 合成的用于 铜-催化的烯酮与二烷基锌的不对称加成反应中的手性 
亚磷酰胺酯配体应用于苯乙烯的不对称硅氢化反应中. 研究结果表明, 催化剂的选择性取决于配体中胺基的结 构. 如采用非手性胺制备的配体 L30a (MonoPhos)仅取 了 55\% ee (表 6, Entry 1). 而二异丙基胺所得的配体 L30b, 仅取得了 $20 \%$ ee (表 6, Entry 2). 同时, 他们将由 $S$-BINOL 与手性胺制备的配体 L31, 用于不对称硅氢化 反应中, 取得了高达 99\%的对映选择性(表 6, Entry 3), 这是迄今为止钯催化苯乙烯取得的最高的对映选择性. 而手性不匹配的配体 L32, 虽然活性与配体 L31 相当, 然而仅取得了 $60 \%$ ee(表 6, Entry 4).

2004 年, 周其林课题组 ${ }^{[51]}$ 从新颢的螺二氢狮骨架 出发合成了一系列亚磷酰胺酯配体. 与 Johannsen 的研 究结果类似, 他们也发现催化剂的活性与选择性取决于 配体中氮原子上的基团. 当配体中胺基部分为二甲氨 基、二异丙基胺基、吡咯烷基时, 所对应的催化剂活性 不高, 且选择性仅为中等. 采用手性匹配的胺与酚制得 的配体 $\mathbf{L 3 3}$, 其对应的催化剂在苯乙烯的不对称硅氢化 反应中不仅活性高, 且取得了高达 97\% ee(表 6, Entry 5 ). 当底物为 2 -氯苯乙烯时, 所得产物的 $e e$ 值为 $99.1 \%$.
高选择性配体 L31 和配体 L33 均由结构对称的且 手性匹配的仲胺制备而来. 李新生等 ${ }^{[52]}$ 报道了由结构 不对称的手性仲胺合成的联二䒺骨架的亚磷酰胺酯配 体 L34, 在苯乙烯类底物的不对称硅氢化反应中取得了 90\% ee (表 6, Entry 6). 这说明，高对映选择性的手性亚 磷酰胺酯配体不一定需要结构对称的手性胺作为原料.

2008 年, 我们组 ${ }^{[53]}$ 报道了树状结构手性亚磷酰胺 酯配体，在铑催化的官能化烯烃如 $\alpha$-脱氢氨基酸酯及芳 基烯酰胺的不对称氢化反应中，取得了优异的催化性 能，体现了十分明显 “树状分子正效应”。然而，我们 ${ }^{[54]}$ 将树状结构配体 L35 应用于钯催化的苯乙烯的不对称 硅氢化反应中, 仅取得了 $5 \%$ ee (表 6, Entry 7). 这说明 增大胺基的位阻可能不利于实现高效的不对称诱导. 于 是, 我们从联二荎骨架的 3,3'取代的手性联二荎酚出发, 采用价廉易得的非手性的二苄胺作为原料，合成了一类 新型的亚磷酰胺酯配体 L36. 通过对配体的结构与催化 性能的研究, 发现 3,3'为芳基的配体所得产物的选择性 较高(表 6 , Entries 8～10). 其中, 3,3'位为 4-甲氧基苯基 的配体 L36c, 取得了 $96 \%$ ee(表 6, Entry 11). 不仅如此,<smiles>[R]N([R])p1oc2ccc3ccccc3c2c2c(ccc3ccccc32)o1</smiles>

L30a $\mathrm{R}=\mathrm{CH}_{3}$ L30b R= $i-\mathrm{Pr}$

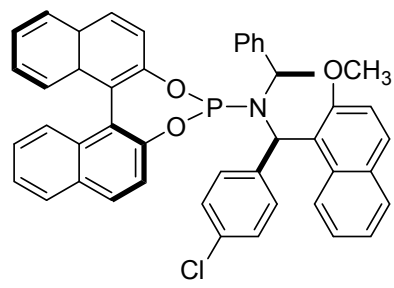

L34

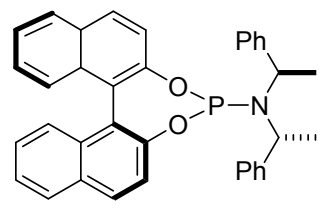

L31<smiles></smiles>

L35<smiles>CC(c1ccccc1)N([C@H](C)c1ccccc1)P(Oc1ccc2ccccc2c1-c1c(Oc2cccc3ccccc23)ccc2ccccc12)c1ccccc1</smiles>

L32<smiles>[R]Cc1cc(OCc2cc(OCc3ccccc3)cc(OCc3ccccc3)c2)cc(OCc2cc(OCc3ccccc3)cc(OCc3ccccc3)c2)c1</smiles><smiles></smiles>

L36a $\mathrm{R}=\mathrm{Ph}$ L36b R = 9-Phenanthryl L36c $\mathrm{R}=4-\mathrm{OCH}_{3}-\mathrm{C}_{6} \mathrm{H}_{4}$<smiles>CC(c1ccccc1)N(C(C)c1ccccc1)P(Oc1ccccc1)c1cccc2c1CCC2</smiles>

L33

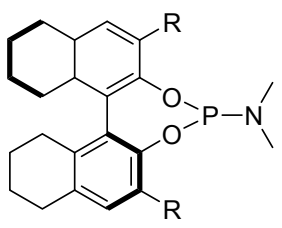

L37a $\mathrm{R}=\mathrm{Ph}$ L37b $\mathrm{R}=4-\mathrm{F}-\mathrm{C}_{6} \mathrm{H}_{4}$ L37c $\mathrm{R}=3,5-\left(\mathrm{CF}_{3}\right)_{2} \mathrm{C}_{6} \mathrm{H}_{3}$

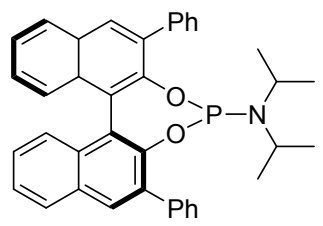

L38<smiles></smiles>

L39

图 5 用于烯烃不对称硅氢化反应的单齿亚磷酰胺酯配体

Figure 5 Chiral monodentate phosphoramidites used in the asymmetric hydrosilylation of alkenes 
表 6 手性亚磷酰胺酯配体用于钯催化的苯乙烯硅氢化反应 ${ }^{a}$ Table 6 Chiral monodentate phosphoramidites used in the asymmetric hydrosilylation of styrene

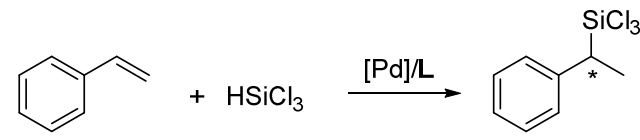

$1 a$

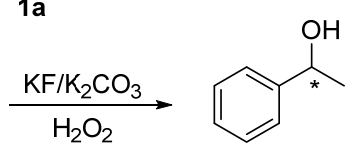

2

\begin{tabular}{|c|c|c|c|c|c|}
\hline Entry & $\mathbf{L}$ & $\begin{array}{c}\text { Catalyst/ } \\
\text { mol\% }\end{array}$ & Condition & $\begin{array}{c}\text { Yield } / \% \\
\text { of } 2\end{array}$ & $\begin{array}{c}e e / \% \text { of } \\
\mathbf{3}\end{array}$ \\
\hline $1^{b}$ & L30a & 1 & r.t., $24 \mathrm{~h}$ & 100 & 55 \\
\hline $2^{b}$ & L30b & 1 & r.t., 24 h & 100 & 20 \\
\hline 3 & L31 & 0.25 & $20{ }^{\circ} \mathrm{C}, 16 \mathrm{~h}$ & 87 & 99 \\
\hline 4 & L32 & 1 & r.t., 24 h & 100 & 60 \\
\hline 5 & L33 & 0.25 & $15 \sim 20{ }^{\circ} \mathrm{C}, 2 \mathrm{~h}$ & 99 & 97 \\
\hline 6 & L34 & 0.125 & r.t., $36 \mathrm{~h}$ & 94 & 90 \\
\hline 7 & L35 & 0.25 & $0{ }^{\circ} \mathrm{C}, 8 \mathrm{~h}$ & 30 & 5 \\
\hline $8^{b}$ & L36a & 0.25 & $0{ }^{\circ} \mathrm{C}, 6 \mathrm{~h}$ & $>95$ & 85 \\
\hline $9^{b}$ & L36a & 0.25 & $-20{ }^{\circ} \mathrm{C}, 8 \mathrm{~h}$ & $>95$ & 92 \\
\hline $10^{b}$ & L36b & 0.25 & $-20{ }^{\circ} \mathrm{C}, 16 \mathrm{~h}$ & $>95$ & 96 \\
\hline $11^{b}$ & L36c & 0.25 & $-20{ }^{\circ} \mathrm{C}, 8 \mathrm{~h}$ & $>95$ & 96 \\
\hline $12^{b}$ & L36a & 0.1 & $0{ }^{\circ} \mathrm{C}, 20 \mathrm{~min}$ & $>95$ & 92 \\
\hline $13^{b}$ & L36a & 0.02 & $0{ }^{\circ} \mathrm{C}, 12 \mathrm{~h}$ & $>95$ & 90 \\
\hline $14^{b}$ & L36a & 0.01 & $0{ }^{\circ} \mathrm{C}, 72 \mathrm{~h}$ & $>95$ & 81 \\
\hline 15 & L37a & 0.25 & r.t., $16 \mathrm{~h}$ & $>99$ & 82.5 \\
\hline 16 & L37b & 0.25 & r.t., $16 \mathrm{~h}$ & $>99$ & 85 \\
\hline 17 & L37c & 0.25 & r.t., $16 \mathrm{~h}$ & $>99$ & 90 \\
\hline 18 & L38 & 0.25 & $-20{ }^{\circ} \mathrm{C}, 72 \mathrm{~h}$ & 99 & 90 \\
\hline
\end{tabular}

${ }^{a}$ All reactions were conducted with $\left[\mathrm{Pd}\left(\mathrm{C}_{3} \mathrm{H}_{5}\right) \mathrm{Cl}\right]_{2}$ as precursor, $[\mathbf{L}] /[\mathrm{Pd}]=2$, solvent free unless otherwise stated. ${ }^{b}[\mathbf{L}] /[\mathrm{Pd}]=1$

该类配体成功地应用于一系列苯乙烯类底物的催化不 对称硅氢化反应中, 取得了高活性(TOF 值高达 3000, 表 6, Entry 12)、高产出率(TON 值高达 10000, 表 6, Entry 14). 这一结果可与由手性胺生成的亚磷酰胺酯配 体的催化体系相脑美.

随后, Beller 等 ${ }^{[55]}$ 报道了由 $3,3^{\prime}$ 位取代的部分氢化的 联二䒺酚和非手性胺制备的手性亚磷酰胺酯配体 L37. 他们详细研究了金属前体、底物与硅烷的比例、配体与 金属的比例、配体的结构及偶合常数 $J(\mathrm{P}, \mathrm{Se})$ 与对映选 择性之间的关系. 在室温、无溶剂条件下，该类配体在 苯乙烯类底物的不对称氢化反应中, 有着很好的催化活 性、选择性及底物广谱性. 最近, Han 等 ${ }^{[56]}$ 也合成了一系 列联二菜 $3,3^{\prime}$ 位取代的亚磷酰胺酯配体, 与我们的研究 结果相似，由非手性胺合成的 3,3'位芳基取代配体 $\mathbf{L 3 8}$ 在苯乙烯的不对称硅氢化反应中, 取得了高达 $90 \%$ ee (表 6, Entry 18). 这些结果都说明高效的亚磷酰胺酯配 体不一定需要手性胺作为原料.

手性亚磷酰胺酯配体在苯乙烯的硅氢化反应中取
得了优异的催化效果, 然而在其他烯烃的不对称硅氢化 反应中却鲜有报道. 2013 年, Hayashi 等 ${ }^{[57]}$ 将由手性胺制 备的配体 $\mathbf{L 3 1}$ 用于 1,3-环己二烯的不对称硅氢化反应 中. 虽然该配体在苯乙烯的硅氢化反应中取得了 $99 \%$ $e e$, 然而在该反应中的 $e e$ 值仅为 $57 \%$ (表 7, Entry 1). 令 人意外的是，采用非手性胺合成的配体 L39 在相同条件 下取得了 $87 \% e e($ 表 7, Entry 2). 这也是迄今为止该底物 在钯催化的不对称硅氢化反应中取得的最高对映选择 性. 受此启发, Han 等 ${ }^{[58]}$ 也将非手性胺制得的配体 $\mathbf{L 3 8}$ 用于 1,3-环己二烯的不对称硅氢化反应中，取得了 $65 \%$ $e e($ 表 7, Entry 4). 与 Hayashi 的结果类似, 手性胺对应的 配体 L40 选择性较差(表 7, Entry 6, 33\% ee).

表 7 手性亚磷酰胺酯配体用于钯催化的 1,3-环己二烯的硅氢 化反应 ${ }^{a}$

Table 7 Chiral monodentate phosphoramidites used in the asymmetric hydrosilylation of 1,3-cychlohexadiene
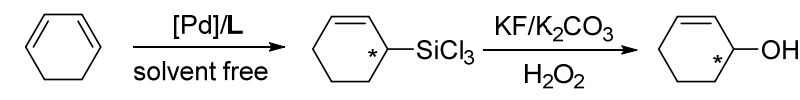

8

10

12

\begin{tabular}{ccccc}
\hline Entry & $\mathbf{L}$ & \multicolumn{1}{c}{ Condition } & Yield/\% of 10a & $e e / \%$ of 12a \\
\hline 1 & $\mathbf{L 3 1}$ & $20{ }^{\circ} \mathrm{C}, 22 \mathrm{~h}$ & 93 & 57 \\
2 & $\mathbf{L 3 9}$ & $20{ }^{\circ} \mathrm{C}, 20 \mathrm{~h}$ & 82 & 87 \\
3 & $\mathbf{L 3 9}$ & $-10{ }^{\circ} \mathrm{C}, 84 \mathrm{~h}$ & 97 & 87 \\
4 & $\mathbf{L 3 8}$ & $20{ }^{\circ} \mathrm{C}, 20 \mathrm{~h}$ & 90 & 65 \\
5 & $\mathbf{L 3 8}$ & $-10{ }^{\circ} \mathrm{C}, 72 \mathrm{~h}$ & 61 & 72 \\
6 & $\mathbf{L 4 0}$ & $20{ }^{\circ} \mathrm{C}, 20 \mathrm{~h}$ & 99 & 33 \\
\hline
\end{tabular}

${ }^{a}$ All reactions were conducted with $\left[\mathrm{Pd}\left(\mathrm{C}_{3} \mathrm{H}_{5}\right) \mathrm{Cl}\right]_{2}$ as precursor, [Ligand]/[Pd] $=2$, solvent free, $1 \mathrm{~mol} \%$ catalyst.

\section{3 手性单齿亚膦酸酯配体在钯催化的烯烃的不 对称硅氢化反应中的应用}

2012 年, Higham 等 ${ }^{[59]}$ 报道了具有两个联二菜单元 的新型手性单齿亚膦酸酯配体. 该配体的合成是以联二 芸骨架的伯膦作为原料与手性联二荎酚(BINOL)反应制 得. 由于配体中含有两个手性单元，他们制备了四种不 同构型的配体，通过核磁以及 $\mathrm{X}$ 射线单晶衍射对钯与配 体的配位行为进行了详细研究. 研究结果表明, 配体中 除了磷原子与金属钯配位外, 荎环上的碳原子也与金属 作用占据剩下的一个配位空轨道. 同时，他们将 4 种不 同构型的配体应用于 $\left[\mathrm{Pd}\left(\mathrm{C}_{3} \mathrm{H}_{5}\right) \mathrm{Cl}\right]_{2}$ 催化的苯乙烯与三 氯硅氢的不对称硅氢化反应中. 其中, 由 $R$ 构型的伯 膦与 $S$ 构型的 BINOL 所得的配体 L41(图 6) 取得了最 高对映选择性 $(80 \% \mathrm{ee})$. 最近，他们组 ${ }^{[60]}$ 报道了类似 结构的配体 L42, 并将其应用于苯乙烯及其衍生物的不 对称硅氢化反应中. 当催化剂前体 $\left[\mathrm{Pd}\left(\mathrm{C}_{3} \mathrm{H}_{5}\right) \mathrm{Cl}\right]_{2}$ 的用 量为 $0.125 \mathrm{~mol} \%$, 配体与金属的比例为 2 时, 室温下, 配体 L42a 取得了 $79 \%$ ee, 配体 L42b 取得了 $80 \%$ ee . 
将反应温度降为 $0{ }^{\circ} \mathrm{C}, 72 \mathrm{~h}$ 后, 配体 $\mathbf{L 4 2 b}$ 取得了 $95 \%$ ee.

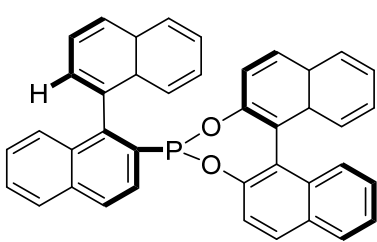

L41

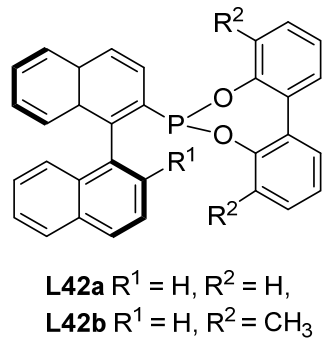

图 6 用于烯烃不对称硅氢化反应的单齿亚膦酸酯配体

Figure 6 Chiral monodentate phophonites used in the asymmetric hydrosilylation of alkenes

\section{4 双膦配体稳定的纳米钯催化剂在不对称硅氢 化反应中的应用}

均相的 BINAP-钯络合物不能催化烯烃的不对称硅 氢化反应. 2003 年, Fujihara 等 ${ }^{[61]}$ 首次合成了手性双膦配 体 BINAP 稳定的直径小于 $2 \mathrm{~nm}$ 的钯纳米颗粒, 并将其 应用于苯乙烯的不对称硅氢化反应中. 研究结果表明, 室温下反应 $5 \mathrm{~h}$, BINAP-钯纳米颗粒使得底物完全转化, 以 $81 \%$ 的收率、 $75 \%$ ee 得到 $S$-苯乙醇. 当反应温度降至 $0{ }^{\circ} \mathrm{C}$ 时, 催化剂活性得以保持, 对映选择性上升至 $95 \%$. 均相的 BINAP-钯络合物在烯烃的不对称硅氢化反应中 没有活性, 这充分说明 BINAP-钯纳米颗粒中特殊的纳 米结构起到了诱导不对称催化反应的结果.

\section{5 总结与展望}

综上所述, 经过三十多年的发展, 随着许多优秀手 性单膦配体的合成与应用，钯催化的烷基烯烃、苯乙烯、 1,3-二烯烃等的不对称硅氢化反应取得了较大的进展, 在整个不对称催化基础研究领域占据非常重要的地位. 尽管烯烃的不对称硅氢化已经发展得比较成熟, 但还有 很大的提升空间. 首先, 开发价廉易得、结构简单、新 颖、可操作性强(不需无水无氧)的, 具有高活性、高区 域选择性、高对映选择性的手性单膦配体仍然是今后研 究的重要内容. 同时, 深入研究烯烃的不对称硅氢化反 应机理, 为设计高效催化体系提供理论依据及指导, 也 是将来需要进一步关注的研究热点. 再次, 发展负载型 手性单膦配体 ${ }^{[62]}$, 借鉴廉价金属 ${ }^{[63,64]}$ 及非金属 ${ }^{[65]}$ 催化 体系的成功经验, 为解决钯的价格昂贵和回收困难导致 难以实现大规模工业化应用的问题. 另外, 相对于不对 称氢化反应, 单膦配体在烯烃的硅氢化反应中底物适用 范围还较窄, 可以尝试将应用面拓展到更多类别更困难 的底物. 我们相信, 随着科研工作者不断深入研究不对 称硅催化反应, 未来钯-单膦催化剂在基础研究及工业
应用方面都将有着更加广阔的前景.

\section{References}

[1] (a) Kiss, G. Chem. Rev. 2001, 101, 3435.

(b) Liebscher, Y. J. Chem. Rev. 2007, 107, 133

(c) Negishi, E. I.; Anastasia, L. Chem. Rev. 2003, 103, 1979.

(d) Ruiz-Castillo, P.; Buchwald, S. L. Chem. Rev. 2016, 116, 12564

[2] (a) Lyons, T. W.; Sanford, M. S. Chem. Rev. 2010, 110, 1147.

(b) Wu, X. F.; Neumann, H.; Beller, M. Chem. Rev. 2013, 113, 1.

(c) Cacchi, S.; Fabrizi, G. Chem. Rev. 2005, 105, 2873.

(d) Li, Z.; Duan, W. L. Chin. J. Org. Chem. 2016, 36, 1805 (in Chinese).

(李振, 段伟良, 有机化学, 2016, 36, 1805.)

(e) Li, J. X.; Zhang, Z. M.; Li, C. S.; Luo, W.; Yang, S. R. Chin. J. Org. Chem. 2015, 35, 2199 (in Chinese).

(李建晓, 张振明, 李春生, 罗维, 杨少容, 有机化学, 2015, 35, 2199.)

[3] (a) Zimmer, R.; Dinesh, C. U.; Nandanan, E.; Khan, F. A. Chem. Rev. 2000, 100, 3067.

(b) Tietze, L. F.; Ila, H.; Bell, H. P. Chem. Rev. 2004, 104, 3453.

[4] McDonald, R. I.; Liu, G. S.; Stahl, S. S. Chem. Rev. 2011, 111, 2981.

[5] (a) Brunner, H.; Becker, R.; Riepl, G. Organometallics 1984, 3, 1354.

(b) Nishiyama, H.; Yamaguchi, S.; Kondo, M.; Itoh, K. J. Org. Chem. 1992, 57, 4306.

[6] Kiso, Y.; Yamamoto, K.; Tamao, K.; Kumada, M. J. Am. Chem. Soc. 1972, 94, 4373

[7] (a) Teichert, J. F.; Feringa, B. L. Angew. Chem., Int. Ed. 2010, 49 , 2486.

(b) Zhang, Z.; Xie, F.; Yang, B.; Yu, H.; Zhang, W. Chin. J. Org. Chem. 2011, 31, 429 (in Chinese).

(张振锋, 谢芳, 杨波, 余焓, 张万斌, 有机化学, 2011, 31, 429.)

(c) Minnaard, A. J.; Feringa, B. L.; Lefort, L.; de Vries, J. G. Acc. Chem. Res. 2007, 40, 1267.

(d) de Vries, A. H. M.; de Vries, J. G. Platinum Metals Rev. 2006, 50,54 .

[8] (a) Feng, X. Q.; Duan, H. F. Chin. J. Org. Chem. 2015, 35, 259 ( in Chinese).

(冯向青, 杜海峰, 有机化学, 2015, 35, 259.)

(b) Rong, J.; Ni, C. F.; Wang, Y. Z.; Kuang, C. W.; Gu, Y. C.; Hu, J. B. Acta Chim. Sinica 2017, 75, 105 (in Chinese).

(荣健，倪传法，王云泽，匡翠文，顾玉诚，胡金波，化学学报， 2017, 75, 105.)

(c) Park, H. S.; Kim, M. Y.; Ahn, H. J.; Han, J. W. Bull. Korean Chem. Soc. 2016, 37, 795.

(d) Ogasawara, M.; Area, S.; Watanabe, S.; Nakajima, K.; Takahashi, T. ACS Catal. 2016, 6, 1308.

[9] (a) Gibson, S. E.; Rudd, M. Adv. Synth. Catal. 2007, 349, 781.

(b) Han, J. W.; Hayashi, T. Tetrahedron: Asymmetry 2010, 21, 2193. (c) Han, J. W.; Hayashi, T. Tetrahedron: Asymmetry 2014, 25, 479.

[10] Liu, Z.; He, X. Proc. Chem. 2006, 18, 1489 (in Chinese). (刘振德，何煦昌，化学进展，2006, 18, 1489.)

[11] Hayashi, T.; Tamao, K.; Katsuro, Y.; Nakae, I.; Kumada, M. Tetrahedron Lett. 1980, 21, 1871.

[12] Uozumi, Y.; Kitayama, K.; Hayashi, T. Tetrahedron: Asymmetry 1993, 4, 2419.

[13] Hayashi, T.; Kabeta, K. Tetrahedron Lett. 1985, 26, 3023.

[14] Hayashi, T.; Matsumoto, Y.; Morikawa, I.; Ito, Y. Tetrahedron: Asymmetry 1990, 1, 151.

[15] Pioda, G.; Togni, A. Tetrahedron: Asymmetry 1998, 9, 3903.

[16] Weber, I.; Jones, G. B. Tetrahedron Lett. 2001, 42, 6983.

[17] Gibson, S. E.; Rendell, J. T.; Rudd, M. Synthesis 2006, 3631.

[18] (a) Pedersen, H. L.; Johannsen, M. Chem. Commun. 1999, 2517. (b) Pedersen, H. L.; Johannsen, M. J. Org. Chem. 2002, 67, 7982.

[19] Ohmura, H.; Matsuhash, H.; Tanaka, M.; Kuroboshi, M.; Hiyama, T.; Hatanaka, Y.; Goda, K. J. Organomet. Chem. 1995, 499, 167. 
[20] Han, J. W.; Tokunaga, N.; Hayashi, T. Helv. Chim. Acta 2002, 85, 3848.

[21] Han, J. W.; Tokunaga, N.; Hayashi, T. J. Am. Chem. Soc. 2001, 123, 12915.

[22] Ogasawara, M.; Ito, A.; Yoshida, K.; Hayashi, T. Organometallics 2006, 25,2715 .

[23] (a) Noyori, R.; Takaya, H. Acc. Chem. Res. 1990, 23, 345.

(b) Berthod, M.; Mignani, G.; Woodward, G.; Lemaire, M. Chem. Rev. 2005, 105, 1801 .

(c) Gao, A.; Ye, Q.; Yu, J.; Liu, W. Chin. J. Org. Chem. 2017, 37, 47 (in Chinese). (高安丽, 叶青松, 余娟, 刘伟平, 有机化学, 2017, 37, 47.)

[24] Uozumi, Y.; Hayashi, T. J. Am. Chem. Soc. 1991, 113, 9887.

[25] Uozumi, Y.; Lee, S. Y.; Hayashi, T. Tetrahedron Lett. 1992, 33, 7185 .

[26] Uozumi, Y.; Hayashi, T. Tetrahedron Lett. 1993, 34, 2335.

[27] Kitayama, K.; Uozumi, Y.; Hayashi, T. J. Chem. Soc., Chem. Commun. 1995, 1533

[28] Hayashi, T.; Hirate, S.; Kitayama, K.; Tsuji, H.; Torii, A.; Uozumi, Y. J. Org. Chem. 2001, 66, 1441.

[29] Hayashi, T.; Niizuma, S.; Kamikawa, T.; Suzuki, N.; Uozumi, Y. J. Am. Chem. Soc. 1995, 117, 9101 .

[30] Bringmann, G.; Wuzik, A.; Breuning, M.; Henschel, P.; Peters, K.; Peters, E. M. Tetrahedron: Asymmetry 1999, 10, 3025.

[31] Kitayama, K.; Tsuji, H.; Uozumi, Y.; Hayashi, T. Tetrahedron Lett. 1996, 37, 4169.

[32] Hayashi, T.; Han, J. W.; Takeda, A.; Tang, J.; Nohmi, K.; Mukaide, K.; Tsuji, H.; Uozumi, Y. Adv. Synth. Catal. 2001, 343, 279.

[33] Han, J. W.; Hayashi, T. Chem. Lett. 2001, 976.

[34] Han, J. W.; Hayashi, T. Tetrahedron: Asymmetry 2002, 13, 325.

[35] Dotta, P.; Kumar, P. G. A.; Pregosin, P. S.; Albinati, A.; Rizzato, S. Organometallics 2004, 23, 2295.

[36] Tschoerner, M.; Pregosin, P.; Albinati, A. Organometallics 1999, 18,670 .

[37] Gladiali, S.; Pulacchini, S.; Fabbri, D.; Manassero, M.; Sansoni, M. Tetrahedron: Asymmetry 1998, 9, 391.

[38] Yasuike, S.; Kawara, S.; Okajima, S.; Seki, H.; Yamaguchi, K.; Kurita, J. Tetrahedron Lett. 2004, 45, 9135.

[39] Ficks, A.; Martinez-Botella, I.; Stewart, B.; Harrington, R. W.; Clegg, W.; Higham, L. J. Chem. Commun. 2011, 47, 8274.

[40] Duclos, M. C.; Singjunla, Y.; Petit, C.; Favre-Réguillon, A.; Jeanneau, E.; Popowycz, F.; Métay, E.; Lemaire, M. Tetrahedron Lett. 2012, 53, 5984

[41] Fer, M. J.; Cinqualbre, J.; Bortoluzzi, J.; Chesse, M.; Leroux, F. R.; Panossian, A. Eur. J. Org. Chem. 2016, 26, 4545.

[42] Kiso, Y.; Yamamoto, K.; Tamao, K.; Kumada, M. J. Organomet. Chem. 1981, 210, 9

[43] (a) Marinetti, A. Tetrahedron Lett. 1994, 35, 5861. (b) Marinetti, A.; Ricard, L. Organometallics 1994, 13, 3956

[44] (a) Okada, T.; Morimoto, T.; Achiwa, K. Chem. Lett. 1990, 999. (b) Sakuraba, S.; Okada, T.; Morimoto, T.; Achiwa, K. Chem. Pharm. Bull. 1995, 43, 927.

[45] Yamomoto, T.; Yamada, T.; Nagata, Y.; Suginome, M. J. Am. Chem. Soc. 2010, 132, 7899.

[46] Hulst, R.; de Vries, N. K.; Feringa, B. L. Tetrahedron: Asymmetry 1994, 5, 699 .

[47] (a) deVries, A. H. M.; Meetsma, A.; Feringa, B. L. Angew. Chem. Int. Ed. 1996, 35, 2375.

(b) Feringa, B. L. Acc. Chem. Res. 2000, 33, 346.

(c) López, F.; Minnaard, A. J.; Feringa, B. L. Acc. Chem. Res. 2007, $40,179$.
[48] (a) Van den Berg, M.; Minnaard, A. J.; Schudd, E. P.; van Esch, J.; de Vries, A. H. M.; de Vries, J. G. J. Am. Chem. Soc. 2000, 122, 11539.

(b) Hu, A. G.; Fu, Y.; Xie, J. H.; Zhou, H.; Wang, L. X.; Zhou, Q. L. Angew. Chem., Int. Ed. 2002, 41, 2348.

(c) Hou, G. H.; Xie, J. H.; Yan, P. C.; Zhou, Q. L. J. Am. Chem. Soc. 2009, 131, 1366

(d) Liu, Y.; Ding, K. L. J. Am. Chem. Soc. 2005, 127, 10488.

[49] (a) Lipowsky, G.; Miller, N.; Helmchen, G. Angew. Chem., Int. Ed. 2004, 43, 4595 .

(b) Ohmura, T.; Hartwig, J. F. J. Am. Chem. Soc. 2002, 124, 15164.

(c) Kiener, C. A.; Shu, C.; Incarvito, C.; Hartwig, J. F. J. Am. Chem. Soc. 2003, 125, 14272 .

[50] Jensen, J. F.; Svendsen, B. Y.; la Cour, T. V.; Pedersen, H. L.; Johannsen, M. J. Am. Chem. Soc. 2002, 124, 4558.

[51] Guo, X. X.; Xie, J. H.; Hou, G. H.; Shi, W. J.; Wang, L. X.; Zhou, Q. L. Tetrahedron: Asymmetry 2004, 15, 2231.

[52] Li, X. S.; Song, J. A.; Xu, D. S.; Kong, L. C. Synthesis 2008, 925.

[53] Zhang, F.; Li, Y.; Li, Z. W.; He, Y. M.; Zhu, S. F.; Fan, Q. H.; Zhou, Q. L. Chem. Commun. 2008, 6048.

[54] Zhang, F.; Fan, Q. H. Org. Biomol. Chem. 2009, 7, 4470.

[55] Junge, K.; Wendt, B.; Enthaler, S.; Beller, M. ChemCatChem 2010 2,453

[56] Park, H. S.; Namgung, S.; Shin, H. M.; Ahn, H. J.; Han, J. W. Bull. Korean Chem. Soc. 2014, 35, 2243.

[57] Park, H. S.; Han, J. W.; Shintani, R.; Hayashi, T. Tetrahedron: Asymmetry 2013, 24, 418.

[58] Park, H. S.; Shin, H. M.; Namgung, S.; Han, J. W. Bull. Korean Chem. Soc. 2014, 35, 2613

[59] Ficks, A.; Hiney, R. M.; Harrington, R. W.; Gilheany, D. G.; Higham, L. J. Dalton Trans. 2012, 41, 3515.

[60] Fleming, J. T.; Ficks, A.; Waddell, P. G.; Paul, G.; Harrington, R. W.; Higham, L. J. Dalton Trans. 2016, 45, 1886.

[61] Tamura, M.; Fujihara, H. J. Am. Chem. Soc. 2003, 125, 15742

[62] (a) Zhong, M. M.; Zhang, X. M.; Zhao, Y. P.; Li, C.; Yang, Q. H. Green Chem. 2015, 17, 1702 .

(b) Shi, L.; Wang, X. W.; Sandoval, C. A.; Li, M. X.; Qi, Q. Y.; Li, Z. T.; Ding, K. L. Angew. Chem., Int. Ed. 2006, 45, 4108

[63] (a) Xu, D. P.; Xiao, W. J.; Peng, J. J.; Li, J. Y.; Bai, Y. Chin. J. Org. Chem. 2014, 34, 2195 (in Chinese).

(徐大鹏，肖文军，彭家建，厉嘉云，白赢，有机化学，2014，34, 2195.)

(b) Chen, L. Z.; Peng, J. J.; Li, J. Y.; Bai, Y.; Qiu, H. Y.; Lai, G. Q. Chin. J. Org. Chem. 2008, 28, 761 (in Chinese)

(陈玲珍, 彭家建, 厉嘉云, 白 赢, 邱化玉, 来国桥, 有机化学, 2008, 28, 761.)

(c) Liu, S.; Peng, J. J.; Li, J. Y.; Bai, Y.; Xiao, W. J.; Lai, G. Q. Chin. J. Org. Chem. 2012, 32, 1827 (in Chinese)

(刘帅，彭家建，厉嘉云，白赢，肖文军，来国桥，有机化学， 2012, 32, 1827.)

[64] (a) Liu, Y. Y.; Zhang, W. B. Chin. J. Org. Chem. 2016, 36, 2249 (in Chinese).

(刘媛媛，张万斌，有机化学, 2016, 36, 2249.)

(b) Li, T. T.; Yu, P.; Lin, J. S.; Zhi, Y. G.; Liu, X. Y. Chin. J. Chem. 2016, 34, 490.

(c) Yang, M. B.; Wang, W. L.; Liu, Y.; Feng, L. J.; Ju, X. X. Chin. J. Chem. 2014, 32, 833 .

(d) Gribble, M. W.; Pirnot, M. T.; Bandar, J. S.; Liu, R. Y.; Buchwald, S. L. J. Am. Chem. Soc. 2017, 139, 2192.

[65] Yang, L.; Lu, W.; Zhou, W.; Zhang, F. Green Chem. 2016, 18, 2941.

(Li, L.; Fan, Y.) 